\title{
ON THE TRANSPORT CAPACITY OF A BROADCAST GAUSSIAN CHANNEL*
}

\author{
ALEX REZNIK ${ }^{\dagger}$ AND SERGIO VERDÚ $\ddagger$
}

\begin{abstract}
The main question addressed in this paper is the problem of maximizing the "transport capacity" of a broadcast network in a Gaussian power-law channel, where by transport capacity we mean a generalization of the bandwidth-distance product as a means of assigning value to the information delivered by a communication network. This problem arises in areas such as cellular network coverage and ad-hoc network design, where the distance or area covered by a transmission is a critical consideration in the system design process.

In the process of addressing this issue we also derive a transport-capacity maximizing resource allocation scheme for a general set of reward and channel penalty functions. The behavior of transport capacity for a very large network of receivers in a Gaussian power-law channel is also examined and a "large-scale" view of the optimal power allocation scheme for a given distance-payoff function is provided.
\end{abstract}

1. Introduction and Problem Setup. In this paper we consider and generalize the notion of transport capacity of a single-transmitter ad-hoc network. Transport capacity was originally defined in the work of Gupta and Kumar [5],[6] as the total bandwidth-distance product that a communication network is capable of supporting in a given area. In this paper we generalize this definition of the transport capacity to include more general payoff functions and consider the resulting "transport capacity" that a broadcast network is capable of delivering in a Gaussian channel. However, in generalizing the notion of the transport-capacity, we still subscribe to the basic premise of [5] and [6]: that in many communication systems the cost of delivering a bit of information increases severely with the distance that the information is to be delivered across. In particular, this is true of wireless communication networks, where the signal quality degrades as a power of the distance that the signal has travelled. Thus, in defining a more general payoff function, we constrain ourselves to payoff functions that reward reliable delivery of information over greater distances.

There are a variety of scenarios to which the model treated in this paper is applicable. One such application is in the coverage capabilities of cellular networks, where the forward link is often modelled as a broadcast network. While users close to the base station often get a higher rate assignment, there is typically a need to provide a minimum quality of service to all the users in a given cell. Understanding how to distributed the available resource (power) efficiently is important in planning of such networks. Another application is in the area of ad-hoc networks, which are, by definition, not planned. Given such an "ad-hoc" configuration, it is important

\footnotetext{
*Received on April 23, 2002; accepted for publication on September 6, 2002. Supported in part by ODDR\&E MURI through the Army Research Office, Grant number DAAD19-00-1-0466

${ }^{\dagger}$ InterDigital Communications Corp., King of Prussia, PA 19406, USA.

${ }^{\ddagger}$ Department of Electrical Engineering, Princeton University, Princeton, NJ 08544, USA, E-mail: $\{$ areznik,verdu\}@princeton.edu
} 
to understand the cost of broadcasting messages from one transmitter to several receivers, and this cost can only be properly evaluated when an appropriate value is assigned for delivery of information over a given distance. In both of these cases the general observations that are made when large-scale networks are considered may prove particularly useful as these permit us to step beyond a specific problem defined by a specific location of receivers and examine the general trends that govern optimal power and rate allocation for a given distance payoff function.

Let us then consider, some area of space, in which a single transmitter is transmitting to $K$ receivers at distances $d_{1}, d_{2}, \ldots, d_{K}$ from this transmitter. Without loss of generality assume $d_{1} \leq d_{2} \leq \cdots \leq d_{K}$. Information is transmitted to the $k^{t h}$ receiver at a rate $R_{k}$ and no common information is transmitted. In this paper we are interested in maximizing the "transport capacity" of this network, which is defined in [5] and [6] as the amount of information transmitted per unit distance (measured in bit-meters or bit-meters per second per Hertz):

$$
T C=\sum_{k=1}^{K} d_{k} R_{k} .
$$

Actually, we are interested in a more general payoff function, where the payoff still depends on the distance over which the information is transmitted, however, the exponent is now a general "distance payoff exponent," which we denote by $\rho$. The only restriction placed on $\rho$ is that the payoff actually increase with distance, thus we require $\rho>0$. The transport capacity is then measured in bit-meters ${ }^{\rho}$ and is given by

$$
T C=\sum_{k=1}^{K} d_{k}^{\rho} R_{k} .
$$

In fact, the main results of the paper are developed in complete generality with very few assumptions on both the payoff function and the function which describes how the signal-to-noise ratio degrades with distance. These results are then applied to the specific problem that we are interested in.

We model the channel as a degraded Gaussian broadcast channel. Thus, in a sense, this is a broadcast channel capacity problem and the solution to this problem for the case of an additive white Gaussian noise channel is well known (Chapter 14 of [3]). However, we are not simply interested in achieving some set of rates, but doing this in a matter which would allow us to transmit "as much information as possible as far as possible." Thus, for example, when we consider transport capacity using the optimal communication scheme we operate at the boundary of the capacity region and choose the set of rates on this boundary that is optimal according to whatever definition of transport capacity happens to be of interest. Our solution turns out to be a special case of the optimal power allocation solution for fading broadcast channels as presented in [8]. However, while [8] only presents an algorithm for determining 
what the optimal power allocation scheme should be in any given case, we give a completely explicit solution. This allows us to obtain insights and make conclusions which would be difficult to make on the basis of the results in [8].

The paper is organized as follows. In Section 2 we summarize results on capacity of the Gaussian broadcast channel which are used throughout the paper.

In Section 3 we consider transport capacity for the information-theoretically optimal communication scheme. We derive the optimal communication strategy. We also use a "very large network" argument to make some general observations about the transport capacity of a broadcast network. In particular, we note that the power control strategy suggested by transport capacity considerations turns out to be counter to the information-theoretic "water-filling" strategy and along the lines of conventional power control strategies used in the forward links of typical cellular communication systems.

For the most common model, where the signal-to-noise ratio at the receiver depends on distance via a power law, we observe that the capacity of a very large network actually grows as the channel gets worse (the exponent of the power law increases). We attribute this phenomenon to a problem which the power law model has at distances close to 0 and are able to provide a simple modification to this model such that the capacity of a very large network does degrade as the channel becomes worse. We re-develop the optimal solution for this modified model.

In Section 4 we consider transport capacity for an orthogonal multiplexing scheme. This accurately models various multiplexing approaches used in practice, most notably FDMA, TDMA and orthogonal CDMA. For convenience we refer to orthogonal multiplexing as TDMA. Again, we derive the optimal communication strategy for both the power law channel and the modified power law channel and compare the resulting transport capacity with that obtained for the optimal communication scheme. In particular, for a large network, we observe that gains in the transport capacity can be achieved using the information-theoretically optimal communication scheme versus an orthogonal multiplexing scheme.

Because the problem considered in this paper is intimately tied to the relative distances between the receivers and the transmitter, we use terminology that reflects this fact. We say that a receiver $a$ is behind receiver $b$ if the distance from $a$ to the transmitter is greater then the distance from $b$ to the transmitter. The words closer, in front, etc. are to be understood in the same context. Finally, we refer to a specific distribution of rates between receivers as a communication strategy, and we seek to find an optimal communication strategy - that is one that maximizes the total transport capacity.

2. Broadcast Capacity Results. The discussion here summarizes the essential results on capacity of broadcast channels. More information can be found in Chapter 14 of [3].

A broadcast channel consists of an input alphabet $\mathcal{H}, K$ output alphabets $\mathcal{Y}_{1}, \mathcal{Y}_{2}$, 
$\ldots, \mathcal{Y}_{K}$ and a probability transition function $p\left(y_{1}, y_{2}, \ldots, y_{k} \mid x\right)$. A broadcast channel is said to be physically degraded if

$$
p\left(y_{1}, y_{2}, \ldots, y_{k} \mid x\right)=p\left(y_{1} \mid x\right) p\left(y_{2} \mid y_{1}\right) \cdots p\left(y_{K} \mid y_{K-1}\right)
$$

for some appropriate ordering of the outputs.

The capacity of broadcast channels has been considered in an early paper by Cover [2]. Bergmans [1] specializes the results of [2] to the case of degraded broadcast channels and proves the coding theorem for such channels. Gallager [4] provides the converse to the coding theorem of [1].

The Gaussian broadcast channel is a degraded broadcast channel and the capacity region of this channel is given by:

$$
\begin{aligned}
& R_{1} \leq C\left(\frac{\alpha_{1} P}{N_{1}}\right) \\
& R_{2} \leq C\left(\frac{\alpha_{2} P}{N_{2}+\alpha_{1} P}\right) \\
& \ldots \\
& R_{k} \leq C\left(\frac{\alpha_{k} P}{N_{k}+P \sum_{i=1}^{k-1} \alpha_{i}}\right) \\
& \ldots \\
& R_{K} \leq C\left(\frac{\alpha_{K} P}{N_{K}+P \sum_{i=1}^{K-1} \alpha_{i}}\right)
\end{aligned}
$$

where $P$ is the total transmitted power; $\alpha_{k}$ is the fraction of the total power allocated to the $k^{\text {th }}$ user, i.e. $0 \leq \alpha_{k} \leq 1$ and $\sum_{k=1}^{K} \alpha_{k}=1$; and $C(\cdot)$ is the "single-user Gaussian capacity function" $C(x) \triangleq \frac{1}{2} \log _{2}(1+x)$.

The region in (3) needs some clarification in terms of its applicability to the problem we are considering. It holds under the following three assumptions:

- We assume that the transmitter is omni-directional - it transmits equally in all directions (in the plane at least). Thus, the signal-to-noise ratio depends only on the distance between the transmitter and the receiver and not on the direction of transmission.

- In the case of a single-dimensional Gaussian power law channel, the ordering imposed by (2) simply implies a distance ordering: the first receiver is the closest (and thus gets the strongest signal), the second receiver is the next one, and so on, the last receiver is the farthest away. Thus, the ordering in (2) and (3) corresponds to the ordering we assumed on distances when we set up the problem. 
We need to add a restriction to the assumptions made in the introduction. We are going to require that $0<d_{1}<d_{2}<\cdots<d_{K}$. This is done without loss of generality. If two or more receivers are equidistant from the transmitter we may consider them as one receiver and find the optimal solution accordingly.

The rate allocated to this "combined receiver" may then be split between the actual receivers in any fashion - the transport capacity remains unchanged.

- While in "real life" the Gaussian (thermal) noise remains constant, but the signal power decreases with distance, it is useful to model the situation in the opposite way - the signal power remains constant, but the noise power increases as the distance from the transmitter increases. The amount of increase of noise power is the same as the "real" decrease in signal power. Specifically, this means that in (3) $N_{k}>N_{k-1}$.

- The results presented in this paper apply to a multi-dimensional Gaussian power law channel if such a channel may be accurately modelled as a degraded physical channel. We expect that the validity of such a model would depend on the specific application.

It is helpful to understand the communication strategy which achieves the region defined by (3). We refer to this strategy as information-theoretically optimal or simply optimal. The strategy is as follows:

- The transmitter generates independent codebooks for each receiver and adds the codewords used at the output. The codewords are "scaled" according to the fraction of the total power allocated to each user.

- The first receiver is close enough to be able to successfully decode all $K$ signals, which it does.

- The second receiver can no longer successfully decode the information intended for the first receiver - this appears to it to be noise. However, it can successfully decode all other signals, which it does.

- In general, the $k^{\text {th }}$ receiver cannot decode signals intended for receivers closer than it is. These appear to the $k^{\text {th }}$ receiver as noise. It can, and does, successfully decode all other signals.

\section{Transport Capacity Using the Optimal Communication Scheme.}

3.1. The general problem. We begin by considering the problem of maximizing the transport capacity using the information-theoretically optimal communication scheme. Specifically, we are interested in defining transport capacity as in (1), with the noise power increasing as a function of distance according to some power law. However, it turns out to be easier to first solve the problem using a much more general setup and then apply the solution which is thus obtained to the specific model of interest. Accordingly, we first define the general problem.

We are given two functions from $[0,+\infty)$ to $[0,+\infty)$ : the reward function $r$ and the noise-to-signal ratio (NSR) function $s$. The reward function specifies the amount of reward associated with transmitting to a distance $d$ and the NSR function defines 
the noise-to-signal ratio at a distance $d$ from the transmitter. The only restriction that we place on these functions at this time is that they be strictly increasing: for $y>x, r(y)>r(x)$ and $s(y)>s(x)$, and that $r(0)=0$ and $s(0) \geq 0$ (thus both $r$ and $s$ are strictly positive on $(0,+\infty))$.

We are given a configuration with $K$ receivers located at distances $d_{1}, \ldots, d_{K}$ with $0<d_{1}<\ldots<d_{K}$. For convenience and clarity we adopt the notation $r_{k} \triangleq r\left(d_{k}\right)$ and $s_{k} \triangleq s\left(d_{k}\right)$.

Re-writing (3) for the $k^{t h}$ receiver we have:

$$
R_{k}=\frac{1}{2} \log _{2}\left(\frac{s_{k}+\beta_{k}}{s_{k}+\beta_{k-1}}\right)
$$

where $s_{k} \equiv \frac{N_{k}}{P}$ as used in (3) and $\beta_{k} \triangleq \sum_{i=1}^{k} \alpha_{i}$ with $\beta_{0} \equiv 0$ and $\beta_{K} \equiv 1$.

We are interested in optimizing

$$
f(\mathbf{b})=\sum_{i=1}^{K} r_{k} R_{k}=\frac{1}{2} \sum_{i=1}^{K} r_{k} \log _{2}\left(\frac{s_{k}+\beta_{k}}{s_{k}+\beta_{k-1}}\right)
$$

where $\mathbf{b} \triangleq\left[\beta_{1}, \ldots, \beta_{K-1}\right]^{T}$ and subject to $\beta_{k} \geq \beta_{k-1}$ for $1 \leq k \leq K$.

We begin by considering the unconstrained optimization problem of simply maximizing (5) for any $\mathbf{b} \in \Re^{K}$. To do this, we have for $1 \leq k \leq K-1$

$$
\frac{\partial f}{\partial \beta_{k}}=\frac{1}{2 \ln 2}\left(\frac{r_{k}}{s_{k}+\beta_{k}}-\frac{r_{k+1}}{s_{k+1}+\beta_{k}}\right)
$$

setting this equal to 0 , we determine that the candidate optimal solution is given by

$$
\beta_{k}^{*}=\frac{s_{k+1} r_{k}-s_{k} r_{k+1}}{r_{k+1}-r_{k}}, \quad 1 \leq k \leq K-1 .
$$

The first item to check is that the solution given in (7) does indeed achieve the maximum. Since $f(\mathbf{b})$ is twice differentiable and $(7)$ defines the unique point at which $\nabla f(\mathbf{b})=0$, it is sufficient to check that the matrix $\nabla^{2} f(\mathbf{b})$ is negative definite at $\mathbf{b}=\mathbf{b}^{*}$.

From (6) we note that $\frac{\partial f}{\partial \beta_{k}}$ depends only on $\beta_{k}$ and therefore $\nabla^{2} f(\mathbf{b})$ is a diagonal matrix for all $\mathbf{b}$. Thus, we simply need to make sure that $\frac{\partial^{2} f\left(\mathbf{b}^{*}\right)}{\partial \beta_{k}^{2}}<0$ for all $1 \leq k \leq$ $K-1$. We have:

$$
\frac{\partial^{2} f}{\partial \beta_{k}^{2}}=\frac{1}{2 \ln 2}\left(-\frac{r_{k}}{\left(s_{k}+\beta_{k}\right)^{2}}+\frac{r_{k+1}}{\left(s_{k+1}+\beta_{k}\right)^{2}}\right) .
$$

Substituting from (7) we get:

$$
\frac{\partial^{2} f\left(\mathbf{b}^{*}\right)}{\partial \beta_{k}^{2}}=\frac{1}{2 \ln 2}\left(\frac{r_{k+1}-r_{k}}{s_{k+1}-s_{k}}\right)^{2}\left(-\frac{1}{r_{k}}+\frac{1}{r_{k+1}}\right)
$$

which is negative because $r_{k}<r_{k+1}$. 
We now substitute (7) into $R_{k}$ and obtain for $1 \leq k \leq K-1$

$$
R_{k}^{*}=\frac{1}{2} \log _{2}\left(\frac{r_{k}-r_{k-1}}{r_{k+1}-r_{k}} \frac{s_{k+1}-s_{k}}{s_{k}-s_{k-1}}\right)
$$

where we took $r_{0} \triangleq r(0)=0$ and $s_{0} \triangleq s(0)$ to avoid having to write a special equation for $k=1$.

From (8), we make two important observations. First, the expression inside the logarithm is always positive, and therefore the optimal solution to the unconstrained problem is always well-defined. Second, if we are to have $R_{k}^{*}>0$, then we must have

$$
\frac{s_{k+1}-s_{k}}{s_{k}-s_{k-1}}>\frac{r_{k+1}-r_{k}}{r_{k}-r_{k-1}} .
$$

Recall that the optimization of $f(\mathbf{b})$ was to be carried out under three constraints: $\beta_{k} \geq 0, \beta_{k} \leq 1$ and $\beta_{k+1} \geq \beta_{k}$ for $1 \leq k \leq K$. At this point we impose only the last of these constraints and find that the unconstrained optimal solution satisfies $\beta_{k}>\beta_{k-1}$ if and only if

$$
\frac{s_{k+1}-s_{k}}{s_{k}-s_{k-1}}>\frac{r_{k+1}-r_{k}}{r_{k}-r_{k-1}} \quad \forall 1 \leq k \leq K-1
$$

which is the same condition as we needed to ensure $R_{k}^{*}>0$.

Let's examine (9) closer. This restriction states that the reward function grows slower than the NSR function, which is to say the channel quality degrades faster with distance than the reward increases. Such a situation is common in many problem and we will, from now on, assume that (9) holds for $r$ and $s$ in addition to the assumptions already made on these functions.

The property (9) may not always be easy to check, however, the following lemma provides a sufficient condition for (9) that is applicable in wide generality.

Lemma 1. Suppose that $r$ and $s$ are both differentiable on $(0,+\infty)$ and that for all $y>x>0$

$$
\frac{s^{\prime}(y)}{s^{\prime}(x)}>\frac{r^{\prime}(y)}{r^{\prime}(x)}
$$

than $r$ and $s$ satisfy (9) for all $0<x<y$.

Proof. Writing (10) for any point in $\left(d_{k}, d_{k+1}\right]$ and some $t \leq d_{k}$ and then integrating over this interval we get:

$$
\frac{\int_{d_{k}}^{d_{k+1}} s^{\prime}(t) d t}{s^{\prime}(t)}>\frac{\int_{d_{k}}^{d_{k+1}} r^{\prime}(t) d t}{r^{\prime}(t)}
$$

which gives us:

$$
\frac{s_{k+1}-s_{k}}{s^{\prime}(t)}>\frac{r_{k+1}-r_{k}}{r^{\prime}(t)}
$$


The above is equivalent to

$$
\frac{s^{\prime}(t)}{s_{k+1}-s_{k}}<\frac{r^{\prime}(t)}{r_{k+1}-r_{k}}
$$

We now write this inequality for any $t \in\left(d_{k-1}, d_{k}\right]$ and then integrate over this interval to get:

$$
\frac{\int_{d_{k-1}}^{d_{k}} s^{\prime}(t) d t}{s_{k+1}-s_{k}}<\frac{\int_{d_{k-1}}^{d_{k}} r^{\prime}(t) d t}{r_{k+1}-r_{k}}
$$

which evaluates to:

$$
\frac{s_{k}-s_{k-1}}{s_{k+1}-s_{k}}<\frac{r_{k}-r_{k-1}}{r_{k+1}-r_{k}}
$$

which is the desired result, inverted.

Returning to the constrained optimization problem we note that (9) is not sufficient to guarantee that the unconstrained solution is optimal for the constrained problem. In fact, there may be some integer $\tilde{K}$ such that $\beta_{k}^{*}<0 \forall k \leq \tilde{K}$ and another integer $\hat{K}$ such that $\beta_{k}^{*}>1 \forall k \geq \hat{K}$. However, it is tempting to state that the transport-capacity optimizing solution involves communicating only to the receivers with indexes between $\tilde{K}$ and $\hat{K}$. This is not quite true, but the actual solution is close to this. To state this more precisely, we start with the following result.

TheOREM 1. There exist two integers $\tilde{K}$ and $\hat{K}, 0 \leq \tilde{K} \leq \hat{K} \leq K$, such that the transport-capacity optimizing communication scheme involves non-zero rate transmission to all receivers with indices $\tilde{K} \leq k \leq \hat{K}$, at most one receiver with index $k<\tilde{K}$, and at most one receiver with index $k>\hat{K}$.

To prove Theorem 1, we need two lemmas which are stated below. The first of these simply couches the solution to the unconstrained optimization problem as a necessary condition for optimality of a communication strategy. The second is a useful inequality.

Lemma 2. Suppose that the constrained optimization problem for $K$ receivers assigns nonzero rate only to the $M$ receivers in the subset $\mathcal{M} \subset\{1, \ldots, K\}$. Then the solution to the unconstrained optimization problem for $\mathcal{M}$ yields the nonzero rates found in the constrained optimization problem.

Proof. Consider the reduced problem with the $K-M$ "inactive" receivers removed. Let us solve the unconstrained optimization problem for the reduced set of receivers. If this solution satisfies the constraints, then all receivers are assigned a positive rate and we are done.

Suppose that this solution does not satisfy the constraints. Since the unconstrained solution represents a unique critical point, we know that the fully constrained optimal configuration must lie on the boundary of the feasible region - which means that at least one of the receivers is being allocated 0 rate. Let $m$ be this receiver, $m \in \mathcal{M}$ and let $R_{m}$ be the rate delivered to receiver $m$. 
Thus, the total transport capacity delivered to the receivers in $\mathcal{M}$ whenever $R_{m}>0$ can always be exceeded by our special configuration in which $R_{m}=0$. This conclusion must hold no matter how many "inactive" receivers are added to the network and where they are placed, since adding inactive receivers has no effect on the transport capacity. In particular, it must hold for our original configuration with the specific $K-M$ inactive receivers we had there. However, this is a contradiction since we assumed that the optimal configuration has $R_{m}>0 \forall m \in \mathcal{M}$.

The next lemma is commonly known as the "log-sum inequality" and the proof maybe found in [7], \#117 or in [3].

LEMMA 3. If $x, y, a$ and $b$ are all strictly positive then

$$
x \log \frac{x}{a}+y \log \frac{y}{b} \geq(x+y) \log \frac{x+y}{a+b}
$$

with equality if and only if $\frac{x}{a}=\frac{y}{b}$.

We now proceed with proof of Theorem 1. We use the terminology beta of a receiver to denote the optimal $\beta$ associated with a receiver in the modified configuration with all inactive receivers removed (i.e. the configuration used in Lemma 2). Thus, the most distant active receiver always has a $\beta$ of 1 . We also refer to the value $\beta_{k}^{*}$ as computed in (7) as the optimal unconstrained $\beta$ of receiver $k$.

We note that as a consequence of Lemma 2, given a set of active receivers with optimal power allocation among them, the $\beta$ of any receiver depends only on the position of that receiver and the next active receiver. Also, the rate allocated to any active receiver depends only on its own position, the position of the active receiver directly in front of it and the position of the active receiver directly behind it. The unconstrained optimal $\beta$ of a receiver depends on its own position and the position of the receiver directly following it, whether active or not. The most distant receiver in a configuration does not have an unconstrained optimal $\beta$ associated with it. Armed with this observation we can prove the following statement.

Consider a configuration with $K$ receivers, $M-1$ of these active $(K>M-1)$ with power allocated optimally among them. In particular, this means that this power allocation satisfies Lemma 2, i.e. it is given by the unconstrained optimal solution to the problem reduced to the $M-1$ active receivers. Now suppose there is an inactive receiver $k$, with reward $r_{k}$ and rate $R_{k}$, such that it is closer to the transmitter than the most distant receiver in $\mathcal{M}$. Suppose if we "activate" this receiver and determine the optimal power allocation for the new configuration of $M$ active receivers this is still given by the unconstrained optimal solution, with all $M$ receivers remaining active, i.e. having strictly positive rates. Then the total transport capacity is always increased by adding such a receiver.

To make this statement precise, let $\mathcal{M}$ be the set of $M-1$ active receivers. Let $m \in \mathcal{M}$ be an active receiver, $r_{m}$ be the reward assigned for transmitting a bit of information to it and $\bar{R}_{m}$ be the rate assigned to it. Thus $\bar{R}_{m}>0 \forall m \in M-1$. Let $\mathcal{F}$ be the feasible region of achievable rates to the receiver in $\mathcal{M}$. We assume that the 
rates have been assigned in an optimal fashion, that is we assume that

$$
\sum_{m \in \mathcal{M}} r_{m} \bar{R}_{m} \geq \sum_{m \in \mathcal{M}} r_{m} R_{m} \quad \forall\left\{R_{m}\right\}_{m \in \mathcal{M}} \in \mathcal{F}
$$

Consider adding another receiver $k$ such that it is closer then the most distant receiver in $\mathcal{M}$. Let $\mathcal{F}^{\prime}$ be the feasible region of achievable rates to the receivers in $\mathcal{M} \bigcup\{k\}$. The optimal configuration for the new set of $M$ active receivers is defined as:

$$
\left\{\hat{R}_{m}\right\}_{m \in \mathcal{M} \cup\{k\}} \triangleq \arg \max \sum_{\left\{R_{m}\right\}_{m \in \mathcal{M} \cup\{k\}} \in \mathcal{F}^{\prime}} r_{m} R_{m} .
$$

We then have the following Lemma.

Lemma 4. If $\hat{R}_{m}>0 \forall m \in \mathcal{M} \bigcup\{k\}$, and thus, by Lemma 2, the optimal solution can still be found by solving the reduced unconstrained optimization problem for just the $M$ receivers of interest, then

$$
\sum_{m \in \mathcal{M} \cup\{k\}} r_{m} \hat{R}_{m} \geq \sum_{m \in \mathcal{M}} r_{m} \bar{R}_{m}
$$

Proof. Since the new receiver cannot be the last receiver, we only have to consider 3 cases: the receiver being added becomes the closest receiver; the receiver being added becomes next-to-the-last receiver; and the receiver being added is somewhere "in the middle," i.e. its not the closest nor the next-to-last. Let's consider these cases one by one. We index only the receivers of interest: i.e. the $M-1$ active receivers and the single receiver being activated. The indexing is always ordered with the receiver closest to the transmitter having index 1.

Suppose the receiver being added is the closest receiver among the $M$ active receivers. Then its index is 1 . The addition of this receiver affects only the rate allocated to itself and receiver 2. Then, we need to show that the contribution from receivers 1 and 2 to the total transport capacity increases when receiver 1 is added. Dropping the irrelevant factor of $\frac{1}{2}$, we need to show

$$
r_{2} \log \left(\frac{r_{2}}{r_{3}-r_{2}} \frac{s_{3}-s_{2}}{s_{2}}\right) \leq r_{2} \log \left(\frac{r_{2}-r_{1}}{r_{3}-r_{2}} \frac{s_{3}-s_{2}}{s_{2}-s_{1}}\right)+r_{1} \log \left(\frac{r_{1}}{r_{2}-r_{1}} \frac{s_{2}-s_{1}}{s_{1}}\right) .
$$

Recalling that logarithm of a product is a sum of logarithms, we can drop common terms from the logarithms multiplying $r_{2}$ on both sides of the inequality. Doing this and re-grouping the right-hand side we get

$$
r_{2} \log \left(\frac{r_{2}}{s_{2}}\right) \leq\left(r_{2}-r_{1}\right) \log \left(\frac{r_{2}-r_{1}}{s_{2}-s_{1}}\right)+r_{1} \log \left(\frac{r_{1}}{s_{1}}\right)
$$

which follows from Lemma 3 since $r_{2}=\left(r_{2}-r_{1}\right)+r_{1}$ and $s_{2}=\left(s_{2}-s_{1}\right)+s_{1}$.

Now suppose that the receiver being added is the next-to-last. Then its index is $M-1$. The addition of this receiver affects only the rate allocated to itself and 
receivers $M-2$ and $M$. Thus, to show that the overall capacity is increased we only need to show that the contribution from these 3 receivers has increased. Thus we need to show that

$$
\begin{gathered}
r_{M-2} \log \left(\frac{r_{M-2}-r_{M-3}}{r_{M}-r_{M-2}} \frac{s_{M}-s_{M-2}}{s_{M-2}-s_{M-3}}\right) \\
+r_{M} \log \left(\frac{r_{M}-r_{M-2}}{r_{M}} \frac{s_{M}+1}{s_{M}-s_{M-2}}\right) \\
\leq \\
r_{M-2} \log \left(\frac{r_{M-2}-r_{M-3}}{r_{M-1}-r_{M-2}} \frac{s_{M-1}-s_{M-2}}{s_{M-2}-s_{M-3}}\right) \\
+r_{M-1} \log \left(\frac{r_{M-1}-r_{M-2}}{r_{M}-r_{M-1}} \frac{s_{M}-s_{M-1}}{s_{M-1}-s_{M-2}}\right) \\
+r_{M} \log \left(\frac{r_{M}-r_{M-1}}{r_{M}} \frac{s_{M}+1}{s_{M}-s_{M-1}}\right) .
\end{gathered}
$$

Re-arranging this and dropping like terms on both sides as we did for the first case we reduce this inequality to

$$
\begin{gathered}
\left(r_{M}-r_{M-2}\right) \log \left(\frac{r_{M}-r_{M-2}}{s_{M}-s_{M-2}}\right) \\
\leq \\
\left(r_{M}-r_{M-1}\right) \log \left(\frac{r_{M}-r_{M-1}}{s_{M}-s_{M-1}}\right)+\left(r_{M-1}-r_{M-2}\right) \log \left(\frac{r_{M-1}-r_{M-2}}{s_{M-1}-s_{M-2}}\right)
\end{gathered}
$$

which, again, follows from Lemma 3.

Finally, let the receiver being added be in the middle. Then only its own rate, the rate of receiver $k-1$ and the rate of receiver $k+1$ are affected. What we need to show is:

$$
\begin{gathered}
r_{k-1} \log \left(\frac{r_{k-1}-r_{k-2}}{r_{k+1}-r_{k-1}} \frac{s_{k+1}-s_{k-1}}{s_{k-1}-s_{k-2}}\right) \\
+r_{k+1} \log \left(\frac{r_{k+1}-r_{k-1}}{r_{k+2}-r_{k+1}} \frac{s_{k+2}-s_{k+1}}{s_{k+1}-s_{k-1}}\right) \\
\leq \\
r_{k-1} \log \left(\frac{r_{k-1}-r_{k-2}}{r_{k}-r_{k-1}} \frac{s_{k}-s_{k-1}}{s_{k-1}-s_{k-2}}\right) \\
+r_{k} \log \left(\frac{r_{k}-r_{k-1}}{r_{k+1}-r_{k}} \frac{s_{k+1}-s_{k}}{s_{k}-s_{k-1}}\right) \\
+r_{k+1} \log \left(\frac{r_{k+1}-r_{k}}{r_{k+2}-r_{k+1}} \frac{s_{k+2}-s_{k+1}}{s_{k+1}-s_{k}}\right) .
\end{gathered}
$$

Re-arranging and dropping like terms on both sides, this becomes

$$
\begin{gathered}
\left(r_{k+1}-r_{k-1}\right) \log \left(\frac{r_{k+1}-r_{k-1}}{s_{k+1}-s_{k-1}}\right) \\
\leq \\
\left(r_{k}-r_{k-1}\right) \log \left(\frac{r_{k}-r_{k-1}}{s_{k}-s_{k-1}}\right)+\left(r_{k+1}-r_{k}\right) \log \left(\frac{r_{k+1}-r_{k}}{s_{k+1}-s_{k}}\right)
\end{gathered}
$$


which, again, follows from Lemma 3.

Lemma 4 provides us with everything we need to prove Theorem 1.

Proof of Theorem 1 (cont.). Given any 2 active receivers, a repeated application of Lemma 4 dictates that all receivers located between them should be added to the configuration, provided that this would not cause a violation of Lemma 2. Since the $\beta$ 's for the receivers are automatically increasing, as we add receivers we only need to worry about de-activating the first currently active receiver (if its $\beta$ becomes negative) and the last currently active receiver (if the $\beta$ of the receiver in front of it exceeds 1 , one of these two receivers may need to be de-activated).

The $\beta$ of the first active receiver depends only on its own position and the position of the receiver directly following it. Thus, to avoid de-activation of the first receiver, we might have a situation where there are inactive receivers between the first and the second receiver.

Similarly, only by changing the position of the next-to-last and last receivers can the situation arise where the $\beta$ of the next-to-last receiver changes. Therefore, we may have a situation where there are inactive receivers between the next-to-last receiver and the last receiver.

Otherwise, the activation of receivers "in the middle" (between the second and next-to-last receivers) cannot make the updated $\beta$ s violate the constraints of the problem. Clearly, the optimal configuration must have these properties as well, or, by Lemma 4, we could improve on it. Therefore, we've proven Theorem 1.

3.2. Edge effects. We now turn our attention to the edge effects. Specifically, we would like to get a handle on how the first and the last receiver affect and are affected by the "middle band" of receivers. Understanding this will allow us to specify the values $\tilde{K}$ and $\hat{K}$ which are used in Theorem 1 . To do this, we need the following result.

Consider two adjacent receivers. The $\beta$ of the closer of the two receivers is increased if it is moved closer to the transmitter, or if the more distant receiver is moved away from the transmitter, or if both occur. This property holds regardless of what other changes are made in the system (i.e. receivers activated, de-activated, moved), as long as no receivers are placed between the two receivers of interest. To state this precisely we note that if we fix the reward and the NSR functions $r(d)$ and $s(d)$ then $\beta_{k}$ is a function of $d_{k}$ and $d_{k+1}$. Then we have the following Lemma.

LEMma 5. $\beta_{k}$ is a strictly monotonically increasing function of the difference $\left(d_{k+1}-d_{k}\right)$.

Proof. We note that the independence of $\beta$ of the closer receiver to changes in the "outer" system follows simply from the fact that $\beta$ of a receiver depends only on its own position and the position of the receiver directly following it. Also, if we prove the lemma for the case when only the closer receiver is moved and the case when only the more distant receiver is moved, the case when both are moved follows 
immediately. Thus, we consider these two cases.

First let the more distant receiver be located at a distance $d$, and the closer receiver moved from distance $y$ to distance $x$, thus $d>y>x$. Then what we need to prove is:

$$
\frac{s(d) r(y)-s(y) r(d)}{r(d)-r(y)}-\frac{s(d) r(x)-s(x) r(x)}{r(d)-r(x)}>0 .
$$

Combining the two fractions, we note that the denominator is always positive and therefore we only need to make sure that the numerator is positive. Multiplying the numerator out, and performing some simplification, we get

$$
s(d) r(y)-s(y) r(d)+s(y) r(x)-s(d) r(x)+s(x) r(d)-s(x) r(y)>0
$$

which holds because it is just another form of inequality (9) which we assume to hold throughout.

Next consider the second case. Let the closer receiver be located at a distance $d$ and the farther receiver be moved from distance $y$ to distance $x$, thus $d<y<x$. Then we need to show that

$$
\frac{s(x) r(d)-s(d) r(x)}{r(x)-r(d)}-\frac{s(y) r(d)-s(d) r(y)}{r(y)-r(d)}>0 .
$$

Again, we combine the the two fractions and concentrate on the numerator, which, after simplification, becomes

$$
s(x) r(y)-s(x) r(d)+s(d) r(x)-s(y) r(x)+s(y) r(d)-s(d) r(y)>0 .
$$

This is again identical to inequality (9).

Using Lemma 5, we can now make precise the values $\tilde{K}$ and $\hat{K}$ in Theorem 1 . Let's begin with $\hat{K}$. Suppose we have an active configuration with $M$ receivers. Suppose there exists a receiver located farther than the last active receiver, such that if it were activated the problem could be re-optimized with conditions of Lemma 2 satisfied (i.e. no receiver is de-activated). Then the transport capacity is increased by doing so. This is stated precisely in the following Lemma.

Lemma 6. Let $\mathcal{M}$ be the initial set of $M$ active receivers and $\bar{R}_{m}$ be the set of optimal rates allocated to these, where, by assumption $\bar{R}_{m}>0 \forall m \in \mathcal{M}$. Let us consider adding a receiver $M+1$ such that $d_{M+1}>d_{m} \forall m \in \mathcal{M}$. Let $\hat{R}_{m}$, $m \in \mathcal{M} \bigcup\{M+1\}$ be the re-optimized set of rates for the receivers in $\mathcal{M} \bigcup\{M+1\}$. If $\hat{R}_{m}>0 \forall m \in \mathcal{M} \bigcup\{M+1\}$ then

$$
\sum_{m \in \mathcal{M} \cup\{M+1\}} r_{m} \hat{R}_{m} \geq \sum_{m \in \mathcal{M}} r_{m} \bar{R}_{m} .
$$

Proof. As usual, let us index the receivers in $\mathcal{M}$ in order from the transmitter. Since the rate delivered to an active receiver depends only on its active neighbors we immediately have $\hat{R}_{m}=\bar{R}_{m}$ for $1 \leq m \leq M-1$. It then remains to show that 


$$
\begin{gathered}
r_{M} \log \left(\frac{r_{M}-r_{M-1}}{r_{M}} \frac{s_{M}+1}{s_{M}-s_{M-1}}\right) \\
\leq \\
r_{M} \log \left(\frac{r_{M}-r_{M-1}}{r_{M+1}-r_{M}} \frac{s_{M+1}-s_{M}}{s_{M}-s_{M-1}}\right)+r_{M+1} \log \left(\frac{r_{M+1}-r_{M}}{r_{M+1}} \frac{s_{M+1}+1}{s_{M+1}-s_{M}}\right) .
\end{gathered}
$$

We re-arrange this into

$r_{M+1} \log \left(\frac{r_{M+1}}{s_{M+1}-1}\right) \leq r_{M} \log \left(\frac{r_{M}}{s_{M}+1}\right)+\left(r_{M+1}-r_{M}\right) \log \left(\frac{r_{M+1}-r_{M}}{s_{M+1}-s_{M}}\right)$

which follows from Lemma 3.

Consider such a choice of a last active receiver that, conditioned on this receiver being active, it is optimal to activate all receivers whose unconstrained optimal $\beta$ (as given by(7)) is less then 1 and greater then some positive value $\epsilon<1$. (We only need $\epsilon$ because we don't yet know what $\tilde{K}$ is).

The question now is can we find a better configuration than this one? We can find a different receiver which also allows activation of all receivers whose unconstrained $\beta$ s are less than 1 and which increases the overall transport capacity. Suppose we select the best among all such receivers. Can we do better still?

We can try selecting a receiver which will force the $\beta$ for the next-to-last receiver to be greater than 1 . To remove this problem we need to remove one of these two receivers (recall: this $\beta$ is not affected by anything else we might do in the system). Removing the more distant receiver will decrease the transport-capacity compared to choosing a last receiver which allows the $\beta$ of the next-to-last receiver to be less than 1 (by Lemma 6). Removing the next-to-last receiver is equivalent to moving it to the position of the receiver in front of it. By Lemma 5, this can only increase $\beta$ further. Then we find that we need to remove the two last receivers, which makes the third-to-last receiver next-to-last and whose $\beta$ has now increased even further. Repeating this process, we eventually find that we are left with only a single active receiver.

The choice is than to either transmit to a single receiver that we chose or to transmit to a lot of the receivers with optimal unconstrained $\beta$ s between 0 and 1 . We can verify that the latter option is always better. To see this, suppose that this is not so. Then the function $f(\mathbf{b})$ defined in (5) attains its highest value in some corner of our constraint region (in $\Re^{M-1}$ ).

The constraint region is defined by $0 \leq \beta \leq 1$ and $\beta_{k} \leq \beta_{k+1}$. It is compact and convex and $f(\mathbf{b})$ is continuous and differentiable and has only one point such that $\frac{\partial f}{\partial \beta_{k}}=0$ for any $\beta_{k}$. Since we have certain receivers whose optimal unconstrained $\beta \mathrm{s}$ are above 0 , we have a local maximum to the constrained problem somewhere, perhaps on the boundary of the constraint region, but not in a corner.

It is impossible for this local maximum to lie inside the constraint region since this would imply the existence of a local minimum or inflection point, which cannot 
be (we can't have $\nabla f=0$ in more than one point). If this local maximum lies on some edge of the constraint region, it has an additional property that stems from the fact that we activated all possible receivers in the neighborhood of this local maximum. Thus, if we travel either along the edge or "into" the constraint region, $f$ decreases at least in some neighborhood of this local maximum. Thus, once again, in order to finally obtain a higher value in some corner, we must have another point where, at least for some $\beta_{k} \mathrm{~s}, \frac{\partial f}{\partial \beta_{k}}=0$ - which is again impossible. Therefore, we have the following result.

\section{THEOREM 2.}

$$
\hat{K}=\max \left\{k: \beta_{k}^{*} \leq 1\right\}
$$

where $\beta_{k}^{*}$ is the unconstrained optimal $\beta$ for receiver $k$ as given by (7). If $\beta_{\hat{K}}^{*}=1$, then no receivers with indices above $\hat{K}$ are active in the optimal configuration. If $\hat{K}<1$, then the index $\hat{k}$ of the single active receiver among those with indexes above $\hat{K}$ is given as follows:

$$
\hat{k}=\operatorname{argmax}_{k>\hat{K}: \hat{\beta}<1} \frac{1}{2} r_{\hat{k}} \log _{2}\left(\frac{r_{\hat{k}}-r_{\hat{K}}}{r_{\hat{k}}} \frac{s_{\hat{k}}+1}{s_{\hat{k}}-s_{\hat{K}}}\right)
$$

where

$$
\hat{\beta}=\frac{s_{\hat{k}} r_{\hat{K}}-s_{\hat{K}} r_{\hat{k}}}{r_{\hat{k}}-r_{\hat{K}}}
$$

Now, let's consider $\tilde{K}$. Let $m$ be the lowest index among all the receivers with positive optimal unconstrained $\beta$ s. We can select to transmit to receiver $m$, or we can select to not transmit to receiver $m$, but to transmit to some single receiver in front of it (whose $\beta$, by Lemma 5 , would also be positive). If we choose to transmit to more than one receiver with index below $m$, then by Theorem 1 , we should also transmit to receiver with index $m-1$. However, in this case we would violate Lemma 2 , since the $\beta$ of receiver $m-1$ must, by supposition, be negative. Thus, we have the following result.

THEOREM 3.

$$
\tilde{K}=1+\min \left\{k: \beta_{k}^{*} \geq 0\right\}
$$

where $\beta_{k}^{*}$ is the unconstrained optimal $\beta$ for receiver $k$ as given by (7). The single receiver with index less than $\tilde{K}$ is then chosen in such a way that the overall transport capacity is maximized.

Let us consider some special cases. First, let the NSR function be such that $s(0)=0$. In that case $\beta_{k}>0$ for all $k$. To see this, place a "receiver" at a distance $d=0$. Call this receiver 0 . Plugging $s(0)=0$ and $r(0)=0$ into (7) we have $\beta_{0}^{*}=0$. Since for all $k>0 \beta_{k}>\beta_{0}$ the result follows. Thus, we have that if $s(0)=0, \tilde{K}=1$ 
and the transport-capacity maximizing communication scheme involves transmission to all receivers with $\beta_{k}^{*}<1$ and possibly one additional receiver.

Next, consider the case when both $s$ and $r$ are differentiable on $(0,+\infty)$. Then we can use Lemma 5 to get a lower bound on the beta of a receiver located at a distance $x$ from the transmitter. This is given by

$$
\beta^{l b}(x)=\lim _{y \rightarrow x} \frac{s(y) r(x)-r(y) s(x)}{r(y)-r(x)}=\frac{s^{\prime}(x) r(x)-s(x) r^{\prime}(x)}{r^{\prime}(x)} .
$$

We define two critical distances. The lower critical distance is defined as $d^{l c}=x$ such that $\beta_{l b}(x)=0$ and the upper critical distance is defined as $d^{u c}=x$ such that $\beta_{l b}(x)=1$. Using (12) we conclude that

$$
\frac{s\left(d^{l c}\right)}{s^{\prime}\left(d^{l c}\right)}=\frac{r\left(d^{l c}\right)}{r^{\prime}\left(d^{l c}\right)}
$$

and

$$
\frac{s\left(d^{u c}\right)+1}{s^{\prime}\left(d^{u c}\right)}=\frac{r\left(d^{u c}\right)}{r^{\prime}\left(d^{u c}\right)} .
$$

Using Lemma 5 we also have the following

LEMMA 7. The transport capacity maximizing communication scheme involves transmission to all receivers with $\beta_{k}^{*} \leq 1$ located at distances between $d^{l c}$ and $d^{u c}$, except, possibly, the one nearest to the transmitter. No more than one receiver located at a distance greater than $d^{u c}$ from the transmitter may be active in the transportcapacity maximizing configuration.

3.3. Application: the power law channel. We now return to the problem we are interested in. Specifically, we consider a physical channel where the signal-to-noise ratio decreases as the $\gamma$ power of the distance. Then the NSR function is given by

$$
s(d)=\frac{N}{P} d^{\gamma}, \quad \gamma>0
$$

where the quantity $\frac{P}{N}$ is referred to as the SNR density of the system and is measured in meter $^{\gamma}$.

In $[5]$ and $[6]$ the reward function is simply the distance $d$. We consider a more general reward function

$$
r(d)=d^{\rho}, \quad \rho>0
$$

the motivation may be that, for example, we are interested not in transmitting data "as far as possible", but in covering "as wide an area as possible", in which case choosing $\rho=2$ is more appropriate than $\rho=1$. Alternatively, we may vary $\rho$ to finetune our model to reflect the particular requirements that we have in mind (reward the users closer in slightly more or slightly less). 
Both $s(d)$ and $r(d)$ are differentiable and therefore we may use Lemma 1 to conclude that (9) holds as long as $\rho<\gamma$. We assume that this is so for the remainder of the discussion.

The optimal unconstrained $\beta$ s are given by

$$
\beta_{k}^{*}=\frac{N}{P} \frac{d_{k+1}^{\rho} d_{k}^{\rho}\left(d_{k+1}^{\gamma-\rho}-d_{k}^{\gamma-\rho}\right)}{d_{k+1}^{\rho}-d_{k}^{\rho}} .
$$

Since $s(0)=0$ in this case, the optimal communication strategy involves transmission to all receivers for which $\beta_{k}^{*}$ as given by (23) is less than 1 and one additional receiver from among the rest. The rate allocated to all the receivers but the last one is given by

$$
R_{k}=\frac{1}{2} \log _{2}\left(\frac{d_{k}^{\rho}-d_{k-1}^{\rho}}{d_{k+1}^{\rho}-d_{k}^{\rho}} \frac{d_{k+1}^{\gamma}-d_{k}^{\gamma}}{d_{k-1}^{\gamma}-d_{k-1}^{\gamma}}\right)
$$

At this point we present some examples of the optimal power allocation.

Consider first a situation with 10 equally spaced receivers, with receiver $k$ located at a distance $k$ from the transmitter. Figures 1 through 6 show the optimal power allocation (the vector $\mathbf{a}=\left[\alpha_{1}, \ldots, \alpha_{10}\right]$ ) and the resulting rates for several SNR densities $\left(\frac{P}{N}\right)$ at the transmitter. In all of these cases we take $\rho=1$ and $\gamma=2$.

Figures 7 through 10 illustrate the situation when the receivers are "bunched up," so that the distance vector is $\mathbf{d}=[1,2.02,2.04,2.06,2.08,8.02,8.04,8.06,8.08,10]$.

We note from Figures 1 through 6 that the dynamics of the optimal power allocation is in some sense intuitive. The receivers closer to the transmitter get allocated a higher rate while more power is actually allocated to the receivers farther away. Thus, a tradeoff is achieved between the fact that it is cheaper to transmit to receivers closer by, but doing so provides less payoff per bit. The case when $\frac{P}{N}=50 \mathrm{~m}^{2}$ also illustrates the idea of the "upper critical distance" which we introduced at the end of Section 3.2. Namely, there is some distance (here it is somewhere between 6 and 7) beyond which it makes sense to transmit to only a single receiver. The case when $\frac{P}{N}=1000 \mathrm{~m}^{2}$ demonstrates the reverse effect. In this case, there is, in some sense, too much power being transmitted. As a result, we would really like to transmit to receivers far away which do not exist. Thus, a disproportionately large rate and power budget are assigned to the last receiver.

The case illustrated in Figures 7 through 10 highlights another effect. As we pointed out, for the receivers "in the middle block" the rate depends only on the distances between them and their nearest neighbors. Thus, the receivers in the middle of a "bunch" get allocated very small rates, while the receivers at the edges of each bunch get allocated a lot of rate and power.

3.4. Large networks in a power law channel. To get an idea of how the transport capacity of a large broadcast network depends on the transmit signal-tonoise ratio we imagine, instead of a discrete number of receivers, a diffuse distribution 
of receivers in space. Thus, we let the receivers be distributed in space according to some measure $\varphi$, where if $A \subset(0, \infty)$ is a set of distances, $\varphi(A)$ is a (relative) number of receivers located at these distances away from the transmitter. For example, $\varphi((2,3) \cup(4,5))$ is the (relative) number of receivers located at a distance of between 3 and 4 or 4 and 5 away from the transmitter. The $\sigma$-algebra is taken to be the Borel $\sigma$-algebra on $(0,+\infty)$. We also switch to using $x$ to denote distance. This is done to avoid confusion of the distance with the differential.

We require $\varphi$ to be absolutely continuous with respect to the Lebesgue measure $\lambda$ in the Radon-Nikodym sense. Thus, we can define the density function (RadonNikodym derivative of $\varphi$ with respect to the Lebesgue measure) $p(x)$ such that $\varphi(d x)=$ $p(x) \lambda(d x)$, where $p(x)<\infty$ for all $x>0$ and $p(x)>0$.

$p(x)$ should be interpreted very carefully. We emphasize that we are trying to extend what is inherently a discrete problem into a continuous case to gain some additional intuition. As such, $p(x)$ represents the number of receivers in some thin sliver of space. For example, for a one-dimensional plane, $p(x)$ is the number of receivers in a small interval $\Delta x$ around $x$. Clearly, then, the only $p(x)$ that makes sense in this case is $p(x)=1$. Similarly, when considering a two-dimensional situation, the "sliver" $\Delta x$ becomes a thin toroid at a radius $x$ and the only $p(x)$ that makes sense is $p(x)=2 \pi r$. We also note that under such a restriction we would usually have $p(x)>0$ for $x>0$.

Finally, we make an important note. The edge effects present in Theorem 1 disappear in the continuous extension since a single receiver receives at a 0 rate and therefore has no contribution to the transport capacity. Moreover, for a continuous extension it is always optimal to transmit to all the receivers between the lower critical distance and the upper critical distance. Since we have $s(0)=0$ in this model, $d^{l c}=0$. The upper critical distance will be found below.

Using this setup we can proceed. Based on the $\beta_{k}$ values introduced in (23) we define the "cumulative trade-off function" $\beta(x)$ as the proportion of the total power allocated to all the receivers up to the distance $x$. Let the receivers be located at a distance $\Delta x$ away from each other, where $\Delta x$ is a small distance which approaches 0 , then

$$
\begin{aligned}
\beta(x) & \triangleq \lim _{\Delta x \rightarrow 0} \frac{N}{P}\left[\frac{(x+\Delta x)^{\rho} x^{\rho}\left((x+\Delta x)^{\gamma-\rho}-x^{\gamma-\rho}\right)}{(x+\Delta x)^{\rho}-x^{\rho}}\right] \\
& =\lim _{\Delta x \rightarrow 0} \frac{N}{P}\left[\frac{x^{2 \rho}\left((x+\Delta x)^{\gamma-\rho}-x^{\gamma-\rho}\right)}{(x+\Delta x)^{\rho}-x^{\rho}}\right] \\
& =\frac{N}{P} \frac{\gamma-\rho}{\rho} x^{\gamma} .
\end{aligned}
$$

We can now find the upper critical distance by setting $\beta(x)=1$ in (25) and solving for $x$, which results in

$$
d^{u c}=\sqrt[\gamma]{\frac{P}{N} \frac{\rho}{\gamma-\rho}}
$$


Observe from (26) that the critical distance depends only on the properties of the channel - the SNR density and the power law. It is independent of the distribution of receivers.

Next, we define the "tradeoff function" $\alpha(x)$ which is the relative portion of the total power allocated to receivers located approximately $x$ away from the transmitter. This function is the analog of the trade-off values $\alpha_{k}$, used in (3), which determine the proportion of power allocated to receiver $k$ in the discrete case. Then,

$$
\alpha(x) \triangleq \lim _{\Delta x \rightarrow 0} \frac{\beta(x+\Delta x)-\beta(x)}{\Delta x}=\frac{N}{P}(\gamma) \frac{(\gamma-\rho)}{\rho} x^{\gamma-1} .
$$

We note that the tradeoff function represents, in some sense, a power control strategy. This interpretation provides a surprising insight. While the standard information-theoretically optimal power control strategy would involve supplying more power to the users with a better SNR (the "water-filling" solution), our transport-capacity maximizing power control strategy for $\gamma>1$ is to supply more and more power to receivers farther and farther away, until we exhaust the power budget. When the power budget is exhausted we simply give up, abandoning the receivers "too far away to be reached." This strategy is akin to the downlink power control currently employed in many cellular systems.

Finally we proceed to compute the actual transport capacity of this "diffuse" network. If we have a receiver at a distance $x$ and the next receiver is at a distance $\Delta x$, then, recalling that $\beta(x+\Delta x) \approx \beta(x)+\alpha(x) \Delta x$ the rate at which the receiver at $\Delta x$ is receiving at is given by:

$$
\frac{1}{2} \log _{2}\left(1+\frac{\alpha(x) \Delta x P}{N x^{\gamma}+P \beta(x)}\right)
$$

which, using the Taylor expansion about $\Delta x=0$ is approximately equal to

$$
\frac{1}{2 \ln 2} \frac{\alpha(x) P}{N x^{\gamma}+P \beta(x)} \Delta x=\frac{1}{2 \ln 2}(\gamma-\rho) \frac{1}{x} \Delta x .
$$

Then we can define the transport capacity of this diffuse network to be:

$$
T C_{d f}=\int_{0}^{d^{u c}} x^{\rho} \frac{1}{2 \ln 2}(\gamma-\rho) \frac{1}{x} \varphi(d x)=\frac{1}{2 \ln 2}(\gamma-\rho) \int_{0}^{d^{u c}} x^{\rho-1} p(x) d x .
$$

We summarize these results as Theorem 4, below.

TheOREM 4. Consider a "diffuse receiver" network in a power law channel with channel exponent $\gamma$. Let the reward function be $r(x)=x^{\rho}$, with $\rho<\gamma$. Then the cumulative trade-off function $\beta(x)$, the trade-off function $\alpha(x)$ and the upper critical distance $d^{u c}$ are all independent of the receiver distribution and are given by

$$
\beta(x)=\frac{N}{P} \frac{\gamma-\rho}{\rho} x^{\gamma}
$$




$$
\begin{gathered}
\alpha(x)=\frac{N}{P}(\gamma) \frac{(\gamma-\rho)}{\rho} x^{\gamma-1} \\
d^{u c}=\sqrt[\gamma]{\frac{P}{N} \frac{\rho}{\gamma-\rho}} .
\end{gathered}
$$

The total achievable transport capacity is given by

$$
T C_{d f}=\frac{1}{2 \ln 2}(\gamma-\rho) \int_{0}^{d^{u c}} x^{\rho-1} p(x) d x
$$

where $p(x)$ is the receiver density.

We consider some special cases of $(20)$. Let $\rho=1$. For a one-dimensional distribution $(p(x)=1)$ we have:

$$
T C_{d f}=\frac{\gamma-1}{2 \ln 2} \sqrt[\gamma]{\frac{P}{N} \frac{1}{\gamma-1}}
$$

If the receivers are distributed uniformly in an area of space, then we take $p(x)=2 \pi x$ and

$$
T C_{d f}=\frac{\pi(\gamma-1)}{2 \ln 2}\left(\sqrt[\gamma]{\frac{P}{N} \frac{1}{\gamma-1}}\right)^{2} .
$$

Figure 11 shows the optimal transport capacity with $p(x)=1$ and $\rho=1$ for a range of $\gamma \mathrm{s}$ and $S N R$ densities. We note that for a fixed SNR density, the transport capacity is increasing with $\gamma$ (for sufficiently large values of $\gamma$ ). This is very counter-intuitive since a higher $\gamma$ represents a worse channel and one would expect the transport capacity to decrease with $\gamma$. A possible cause of this phenomenon may be that as the distance between the transmitter and the receiver goes to 0 , the SNR actually increases towards infinity and the higher the $\gamma$ the faster it does so. This allows the receivers close to the transmitter to support a very large rate with little power usage and causes the overall transport capacity to increase with $\gamma$.

This is clearly a fault in the model. It is a consequence of the fact that the powerlaw NSR function approaches 0 as the distance approaches 0 and the rate at which it does so increases with $\gamma$. One way to correct this is to assume, as is done in [6], that no receivers are located within some minimal distance $d_{\min }$ of the transmitter. Another method is to adjust the power-law model so that the SNR at the transmitter is a fixed parameter $\frac{P}{\widetilde{N}}$, but at appreciable distance from the transmitter it still follows the power law. We consider one such channel model in the next sub-section.

3.5. Modified power law channel. Let us define a new channel model so that the channel penalty function is given by

$$
s(d)=\frac{N}{P} d^{\gamma}+\frac{\widetilde{N}}{P}
$$


This changes our results slightly, but not significantly. The optimal unconstrained $\beta$ s are now given by

$$
\beta_{k}^{*}=\frac{N}{P} \frac{d_{k+1}^{\rho} d_{k}^{\rho}\left(d_{k+1}^{\gamma-\rho}-d_{k}^{\gamma-\rho}\right)}{d_{k+1}^{\rho}-d_{k}^{\rho}}-\frac{\tilde{N}}{P}
$$

The rate allocated to the "middle" receivers is the same as in the previous case - it still depends neither on the SNR density $\left(\frac{P}{N}\right)$ nor on the SNR at the transmitter $\left(\frac{P}{\widetilde{N}}\right)$.

We summarize the main results for the modified power law channel in Theorem 5. The proof of this theorem involves replicating the steps we went through for the power law channel and is omitted here.

TheOREM 5. For the modified power law channel, the cumulative tradeoff function, the tradeoff function and the lower and upper critical distances are independent of the receiver distribution.

The cumulative tradeoff function is given by

$$
\beta(x)=\frac{N}{P} \frac{\gamma-\rho}{\rho} x^{\gamma}-\frac{\widetilde{N}}{P} .
$$

The tradeoff function $\alpha(x)$ is identical to that of the power law channel as specified in (27).

The lower critical distance is no longer 0 since $s(0)>0$ and is given by

$$
d^{l c}=\sqrt[\gamma]{\frac{\widetilde{N}}{P} \frac{P}{N} \frac{\rho}{\gamma-\rho}} .
$$

The upper critical distance is given by

$$
d^{u c}=\sqrt[\gamma]{\left(1+\frac{\tilde{N}}{P}\right) \frac{P}{N} \frac{\rho}{\gamma-\rho}} .
$$

Finally, the diffuse network capacity under the modified model is

$$
T C_{d f}^{m}=\frac{1}{2 \ln 2}(\gamma-\rho) \int_{d^{l c}}^{d^{u c}} x^{\rho-1} p(x) d x .
$$

For $p(x)=1$ and $\rho=1(33)$ evaluates to

$$
T C_{d f}^{m}=\frac{\gamma-1}{2 \ln 2}\left(\sqrt[\gamma]{1+\frac{\tilde{N}}{P}}-\sqrt[\gamma]{\frac{\tilde{N}}{P}}\right) \sqrt[\gamma]{\frac{P}{N} \frac{1}{\gamma-1}}
$$

which is just the diffuse transport capacity for the original model, multiplied by the factor $\left(\sqrt{1+\frac{\widetilde{N}}{P}}-\sqrt[\gamma]{\frac{\widetilde{N}}{P}}\right)$. This factor goes to 0 as $\gamma \rightarrow \infty$, and the overall expression 
does have a finite limit as $\gamma \rightarrow \infty$. To evaluate this limit we re-write (34) as

$$
T C_{d f}^{m}=\frac{1}{2 \ln 2} \sqrt[\gamma]{\frac{P}{N}}\left(\sqrt[\gamma]{1+\frac{\widetilde{N}}{P}}-\sqrt[\gamma]{\frac{\widetilde{N}}{P}}\right)(\gamma-1)^{\frac{\gamma-1}{\gamma}}
$$

Since for all $\frac{P}{N}>0, \lim _{\gamma \rightarrow \infty} \sqrt[\gamma]{\frac{P}{N}} \rightarrow 1$, we have

$$
\begin{aligned}
\lim _{\gamma \rightarrow \infty} T C_{d f}^{m} & =\frac{1}{2 \ln 2} \lim _{\gamma \rightarrow \infty} \frac{\sqrt[\gamma]{1+\frac{\widetilde{N}}{P}}-\sqrt[\gamma]{\frac{\widetilde{N}}{P}}}{(\gamma-1)-\frac{\gamma-1}{\gamma}} \\
& =\frac{1}{2 \ln 2} \lim _{a \rightarrow 0} \frac{\left(1+\frac{\widetilde{N}}{P}\right)^{a}-\left(\frac{\widetilde{N}}{P}\right)^{a}}{\left(\frac{1-a}{a}\right)^{a-1}}
\end{aligned}
$$

where we made the substitution $a=\frac{1}{\gamma}$.

The limit in (35) cannot be evaluated directly or using a simple application of L'Hópital's rule. However, we can evaluate it by considering the behavior of the numerator for very small values of $a$. We would like to approximate the function $(1+x)^{a}-x^{a}$ for $a$ slightly above 0 . To do this, we write, $y^{a}=e^{a \ln y}$ and recalling the Taylor series expansion for $e^{z}$ around $z=0$, we have as $a \rightarrow 0$

$$
(1+x)^{a}=e^{a \ln (1+x)} \approx 1+a \ln (1+x)
$$

and

$$
x^{a}=e^{a \ln x} \approx 1+a \ln x .
$$

Therefore, as $a \rightarrow 0$ we have in the numerator

$$
\left(1+\frac{\widetilde{N}}{P}\right)^{a}-\left(\frac{\widetilde{N}}{P}\right)^{a} \approx a\left(\ln \left(1+\frac{\tilde{N}}{P}\right)-\ln \left(\frac{\widetilde{N}}{P}\right)\right) .
$$

Substituting (36) back into (35) we have

$$
\begin{aligned}
\lim _{\gamma \rightarrow \infty} T C_{d f}^{m} & =\frac{1}{2 \ln 2} \lim _{a \rightarrow 0} \frac{a\left(\ln \left(1+\frac{\widetilde{N}}{P}\right)-\ln \left(\frac{\widetilde{N}}{P}\right)\right)}{(1-a)^{1-a} \cdot a^{1-a}} \\
& =\frac{1}{2 \ln 2} \lim _{a \rightarrow 0} \frac{\ln \left(1+\frac{\widetilde{N}}{P}\right)-\ln \left(\frac{\widetilde{N}}{P}\right)}{(1-a)^{1-a} \cdot a^{-a}} .
\end{aligned}
$$

Since both quantities in the denominator approach 1 as $a$ approaches 0 , we conclude 
that

$$
\begin{aligned}
\lim _{\gamma \rightarrow \infty} T C_{d f}^{m} & =\frac{1}{2 \ln 2}\left(\ln \left(1+\frac{\tilde{N}}{P}\right)-\ln \left(\frac{\tilde{N}}{P}\right)\right) \\
& =\frac{1}{2}\left(\log _{2}\left(1+\frac{\widetilde{N}}{P}\right)-\log _{2}\left(\frac{\widetilde{N}}{P}\right)\right) \\
& =\frac{1}{2} \log _{2}\left(1+\frac{P}{\widetilde{N}}\right) .
\end{aligned}
$$

Thus, we conclude that as $\gamma \rightarrow \infty$ the transport capacity is simply the capacity of a single-user Gaussian channel with the signal-to-noise ratio equal to the signal-tonoise ratio at the transmitter.

The optimal transport capacity under the modified power law channel for $p(x)=$ 1, $\rho=1$ and $\frac{P}{N}=100$ is shown in Figure 12. We observe that the behavior for small $\gamma$ is very similar to the standard power law channel, as shown in Figure 11. However, as $\gamma$ increases, the surface levels off to a finite value, which depends on the ratio $\frac{P}{\widetilde{N}}$. We note that, as a function of $\gamma$, the optimal transport capacity still attains a minimum at about 2 . This is once again counter-intuitive since one would expect the transport capacity to decrease monotonically with $\gamma$. The explanation, most likely, is that our simple modification does not model the actual situation near the transmitter sufficiently well and a better model is still required.

The result (37) has been obtained for the case when $\rho=1$ and for the "1dimensional uniform distribution" $p(x)=1$. We can generalize it to a general $\rho$ and a general distribution, provided that the density function $p(x)$ is continuous (that is, the distribution itself is actually diffuse). To do so, we return to (33) and note that as $\gamma \rightarrow \infty$, both the upper and the lower limit of the integral in (33) approach 1 . Then, as long as $p(x)$ is continuous,

$$
\begin{aligned}
\lim _{\gamma \rightarrow \infty} T C_{d f}^{m} & =\frac{1}{2 \ln 2} \lim _{\gamma \rightarrow \infty}(\gamma-\rho)\left(1^{\rho-1} p(1)\right) \\
& \times\left[\sqrt[\gamma]{\left(1+\frac{\tilde{N}}{P}\right) \frac{P}{N} \frac{\rho}{\gamma-\rho}}-\sqrt[\gamma]{\left(\frac{\tilde{N}}{P}\right) \frac{P}{N} \frac{\rho}{\gamma-\rho}}\right] \\
& =\frac{p(1)}{2 \ln 2} \lim _{\gamma \rightarrow \infty}\left(\sqrt[\gamma]{\rho \frac{P}{N}}\right)\left(\sqrt[\gamma]{1+\frac{\tilde{N}}{P}}-\sqrt[\gamma]{\frac{\tilde{N}}{P}}\right)(\gamma-\rho)^{\frac{\gamma-1}{\gamma}}
\end{aligned}
$$

The last line of $(38)$ can be evaluated by noting that $\lim _{\gamma \rightarrow \infty} \sqrt[\gamma]{\rho \frac{P}{N}}=1$ and then substituting $a=\frac{1}{\gamma}$. The resulting limit as $a \rightarrow 0$ is very similar to that in (35) - the only difference is that the base of the denominator exponent is $\frac{1-a \rho}{a}$. The result, once the limit is computed, is the same as in (37) and we conclude that

$$
T C_{d f}^{m}=\frac{p(1)}{2} \log _{2}\left(1+\frac{P}{\widetilde{N}}\right) .
$$


4. TDMA Broadcast Capacity. In this section we consider the problem of maximizing the transport capacity of a single-transmitter Gaussian "ad-hoc" network when the transmitter employs TDMA to communicate with the receivers. We begin again with the most general case. The setup in this case can be made even more general then for the information-theoretically optimal communication scheme, and we consider it in the most general case.

We are given a reward function $r(d)$ for communicating with a receiver at a distance $d$ and a rate function $R(d)$ which determines the maximum rate which can be transmitted to a receiver located at a distance $d$ from the transmitter (the singleuser capacity of the channel). We choose to transmit to the receiver $k$ an $\alpha_{k}$ portion of the time, where $\alpha_{k} \geq 0 \forall 1 \leq k \leq K$ and $\sum_{k=1}^{K} \alpha_{k}=1$. The transport capacity of this communication is given by

$$
f(\mathbf{a})=\sum_{k=1}^{K} \alpha_{k} r\left(d_{k}\right) R\left(d_{k}\right)
$$

where $\mathbf{a} \triangleq\left[\alpha_{1}, \ldots, \alpha_{K}\right]^{T}$. We would like to find a value of a which maximizes $f(\mathbf{a})$.

Since $f(\mathbf{a})$ is a linear function of $\mathbf{a}$ (both $r$ and $R$ are independent of $\mathbf{a}$ ), in order to maximize it we simply need to allocate all the transmission rate to the receiver which provides us with the maximum possible payoff. Thus, we have the following result.

THEOREM 6. The transport capacity of a TDMA broadcast network is maximized by transmitting exclusively to the receiver which provides the maximum payoff. The maximum transport capacity is then given by

$$
\overline{T C}=\max _{1 \leq k \leq K} r\left(d_{k}\right) R\left(d_{K}\right)
$$

provided that the expression is well-defined.

We note here, that except for the requirement that (41) be well defined, Theorem 6 places no requirements on the structure of the problem. We require neither that $r(d)$ nor that $R(d)$ be monotonic, we do not require the channel to be degraded, we do not even require that receivers use the same alphabet. To put Theorem 6 another way, we have a choice of $K$ single-user channels and use the one that maximizes our objective function.

One effective way to compare the transport capacities in the two cases: TDMA and information-theoretically optimal communication - is to consider very large networks. In this case, it is likely that there is a receiver arbitrarily close to a point where $r(d) R(d)$ achieves its global maximum and thus the optimum transport capacity of a "diffuse" network utilizing TDMA is simply given by

$$
\max r(d) R(d)
$$


We note that, provided the distribution density function is strictly positive, the optimum transport capacity of a "diffuse" network utilizing TDMA does not depend on the distribution of the receivers.

This is where the care with which we identified valid diffuse receiver distributions in Section 3 becomes important. We would certainly expect that the optimum transport capacity of a network utilizing TDMA should be no greater then the capacity of a network utilizing the optimal communication scheme. However, had we defined the diffuse receiver distribution "carelessly", we would have found that this is not so. Consider a distribution $p(x)=k$ where $k$ is an arbitrary strictly positive constant. In Section 3 we restrict $k$ to be exactly 1 . If we remove this restriction, we can certainly choose a low enough value of $k$ to produce an arbitrarily low diffuse optimal transport capacity for the optimal communication scheme. The diffuse optimal transport capacity for TDMA, since it does not depend on the distribution, would not change. Thus, for some low enough $k$, we would find that the diffuse optimal transport capacity is greater for TDMA then for the optimal communication scheme!

Returning now to the specific models of interest to us, we find that, for the $\rho$ power reward function and a power law channel, the diffuse transport capacity for TDMA is given by

$$
\overline{T C}_{d f}=\max _{d \geq 0} d^{\rho} C\left(\frac{P}{N d^{\gamma}}\right)
$$

and for the modified power law channel it is given by

$$
\overline{T C}_{d f}^{m}=\max _{d \geq 0} d^{\rho} C\left(\frac{P}{N d^{\gamma}+\widetilde{N}}\right) .
$$

We note that for the power law channel $\lim _{\gamma \rightarrow \infty} \overline{T C}_{d f}$ is infinite, as it is for the optimal communication scheme. To prove this, consider an arbitrary large positive number $L$. Pick a value of $d>0$ such that $d \ll 1$. While the value of $d^{\rho}$ is very small, it is fixed. However, by choosing a large enough value of $\gamma$, the value of $\log _{2}\left(1+\frac{P}{N d^{\gamma}}\right)$ may be made large enough to exceed $L$. Thus, for every there exists a $d>0$ such that $\lim _{\gamma \rightarrow \infty} d^{\rho} C\left(\frac{P}{N d^{\gamma}}\right)>L$ and therefore $\lim _{\gamma \rightarrow \infty} \max _{d \geq 0} d^{\rho} C\left(\frac{P}{N d^{\gamma}}\right)=\infty$.

For the modified power law channel taking the limit is much more difficult, however we can construct a loose argument for what the limit should be as follows. For $d>1$, as $\gamma \rightarrow \infty$, the argument of the $C$ function goes to 1 , and therefore the transport capacity goes to 0 . For all $d<1$, the contribution from $N d^{\gamma}$ to the denominator decreases to 0 as $\gamma \rightarrow \infty$ and therefore the transport capacity is given by $\sup _{d \in(0,1)} d^{\rho} C\left(1+\frac{P}{\widetilde{N}}\right)=C\left(1+\frac{P}{\widetilde{N}}\right)$, which is the same as the limit of the transport capacity using the optimal communication scheme and under the uniform (1dimensional) distribution.

Another explanation for this result is that the lower and upper critical distances for the optimal communication scheme converge to 1 as $\gamma \rightarrow \infty$. Thus, in the limit, the optimal strategy for the optimal communication scheme is the same as for TDMA - communicate to a single receiver. Therefore put forward the following Lemma. 
Lemma 8. For any $\rho>0$ and any $\frac{P}{N}>0$

$$
\lim _{\gamma \rightarrow \infty} \max _{d>0} \frac{1}{2} d^{\rho} \log _{2}\left(1+\frac{P}{N d^{\gamma}}\right)=+\infty
$$

and

$$
\lim _{\gamma \rightarrow \infty} \max _{d>0} \frac{1}{2} d^{\rho} \log _{2}\left(1+\frac{P}{N d^{\gamma}+\widetilde{N}}\right)=\frac{1}{2} \log _{2}\left(1+\frac{P}{\widetilde{N}}\right)
$$

The optimal TDMA transport capacity as a function of $\gamma$ and $\frac{P}{N}$ is shown in Figure 13 for the usual power law channel and Figure 14 for the modified power law channel. In both cases we use the same parameters as were used to produce Figures 11 and 12: $p(x)=1, \rho=1, \frac{P}{\widetilde{N}}$.

It is interesting to note that the overall shape of the TDMA curves is very similar to the the curves for the optimal communication scheme. For the power law channel, the optimal transport capacity increases to infinity with $\gamma$ and it levels of with $\gamma$ for the modified channel. This is in line with our expectations.

5. Conclusions. In the preceding sections we provided a comprehensive analysis of optimum resource allocation for the problem of optimizing a general cost function in a Gaussian broadcast channel which degrades as a function of distance. We showed that the optimum resource allocation scheme involves activating all the receivers located within some appropriate range of distances and, additionally, at most one more receiver both before and after this band. These two edge receivers are needed to pick up "left-over" resources at each end of the main band.

For the specific case of the power-law channel and a power-law reward function we extended our analysis to very large networks. We were able to derive the general transport-capacity-maximizing power control strategy for such networks.

Through the large-network analysis we were also able to identify the problem which the power law channel suffers at distance close to 0 . We suggested a possible modification to the channel penalty function that partially corrects this problem, however even this model may require further improvements.

Finally, we were able to demonstrate the differences which result if an orthogonalmultiplexing scheme like TDMA is used instead of an information-theoretically optimal communication scheme. In this case, not only does the resulting transport capacity suffer significantly, but it is no longer optimal to transmit to any receiver, except the most favorably located one.

The developments in this paper are to be considered a small step towards understanding the issues involved in planning for and communicating in generalized wireless networks. The quality of transmission in such networks depends greatly on the distance. Thus a transport-capacity-like figure of merit, which provides higher rewards for transmitting farther, might often be more appropriate for design of such networks then a plain capacity per user or total capacity. 
However, this work addresses only a very special case of the general network and a very special question - how to allocate resources in a most effective manner. Further investigations in this area must also rely on information theoretic results, such as capacities of relay networks and networks with multiple transmitters and receiver. The available results in this area are limited.

A severe limitation of this work is due to the nature of the degraded physical channel model, which we assume. This makes it difficult for us to develop a model which takes into account non-omnidirectional antenna and where the results would reflect, at least in some sense, not just the relative distances between the transmitter and the receivers but the relative positions of the receivers in space. Additionally, the

model considered here does not incorporate fading, which is an important feature of most wireless channels.

\section{REFERENCES}

[1] Patrick P. Bergmans, Random coding theorem for broadcast channels with degraded components, IEEE Transactions on Information Theory, IT-19, pp. 197-207, March 1973.

[2] Thomas M. Cover, Broadcast channels, IEEE Transactions on Information Theory, IT-18, pp. 2-14, January 1972.

[3] Thomas M. Cover and Joy A. Thomas, Elements of Information Theory, John Wiley \& Sons, Inc., New York, 1991.

[4] Robert G. Gallager, Capacity and coding for degraded broadcast channels, Problemy Peredachi Informatsii, 10:3(1974), pp. 3-14.

[5] Piyush Gupta, Design and Performance Analysis of Wireless Networks. PhD thesis, University of Illinois at Urbana-Champaign, Urbana, Illinois, 2000.

[6] Piyush Gupta and P. R. Kumar, The capacity of wireless networks, IEEE Transactions on Information Theory, IT-46, pp. 388-404, March 2000.

[7] G. Hardy, J. E. Littlewood, and G. Pólya, Inequalities, Cambridge University Press, Cambridge, United Kingdom, second edition, 1952.

[8] Lifang Li and Andrea J. Goldsmith, Capacity and optimal resource allocation for fading broadcast channels - part I: Ergodic capacity, IEEE Transactions on Information Theory, IT-47, pp. 1083-1102, March 2001. 


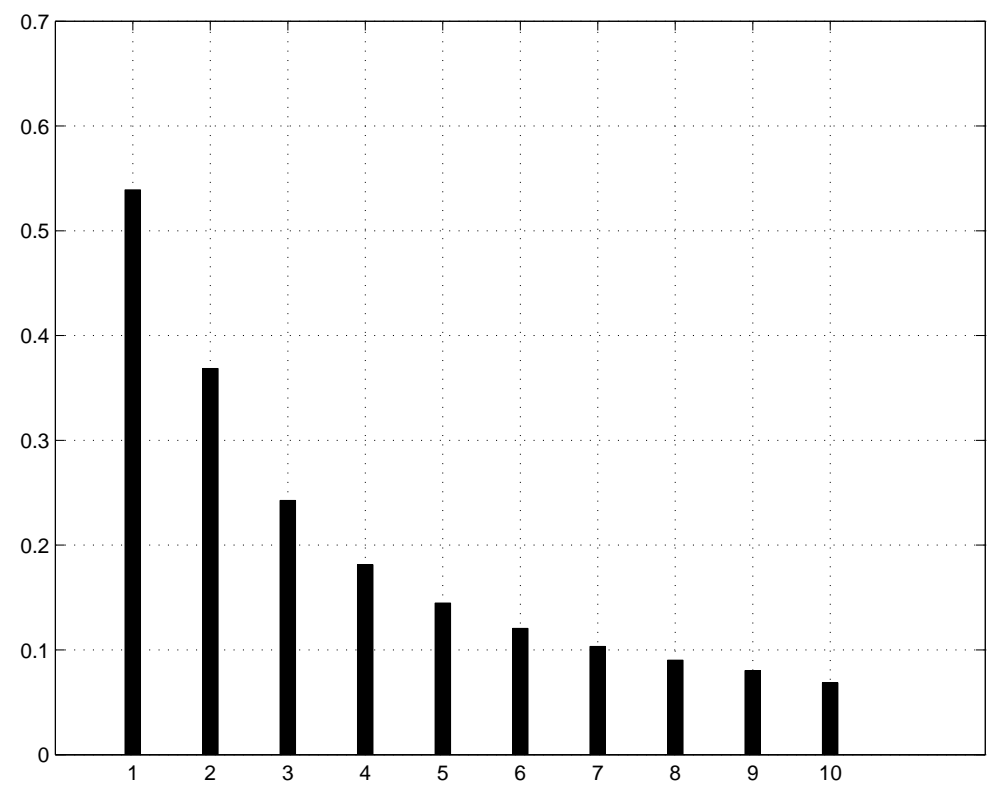

FIG. 1. Rates for 10 uniformly spaced receivers with $S N R$ density $=100 \mathrm{~m}^{2}$.

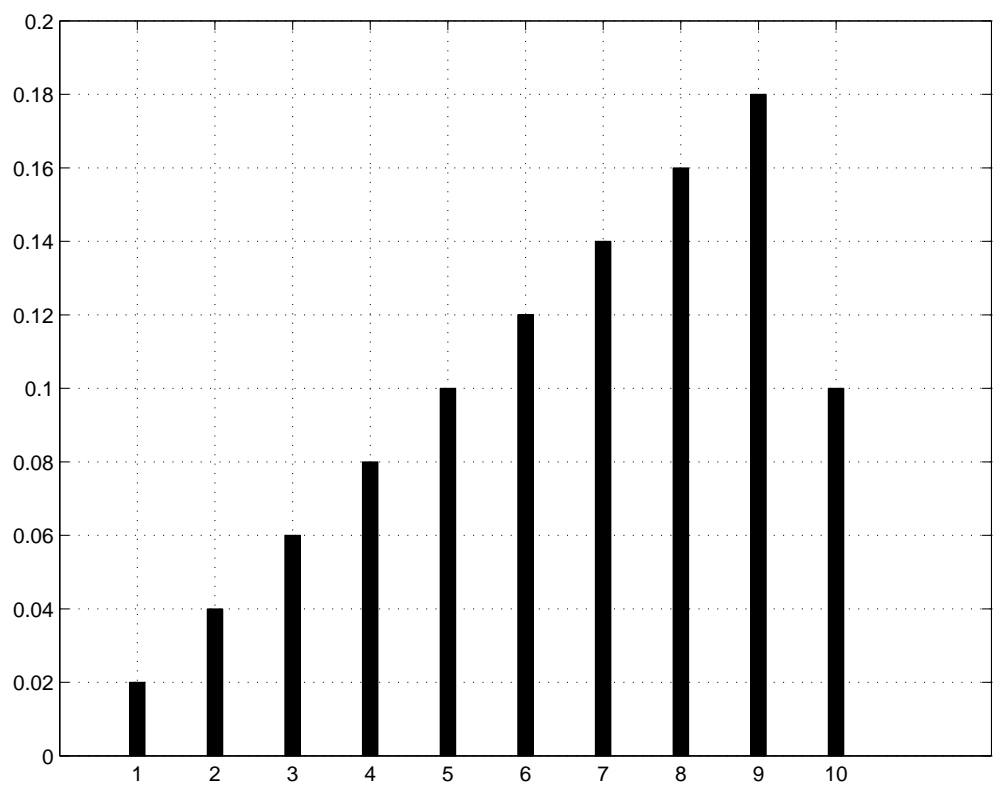

FIG. 2. Power allocation for 10 uniformly spaced receivers with $S N R$ density $=100 \mathrm{~m}^{2}$. 


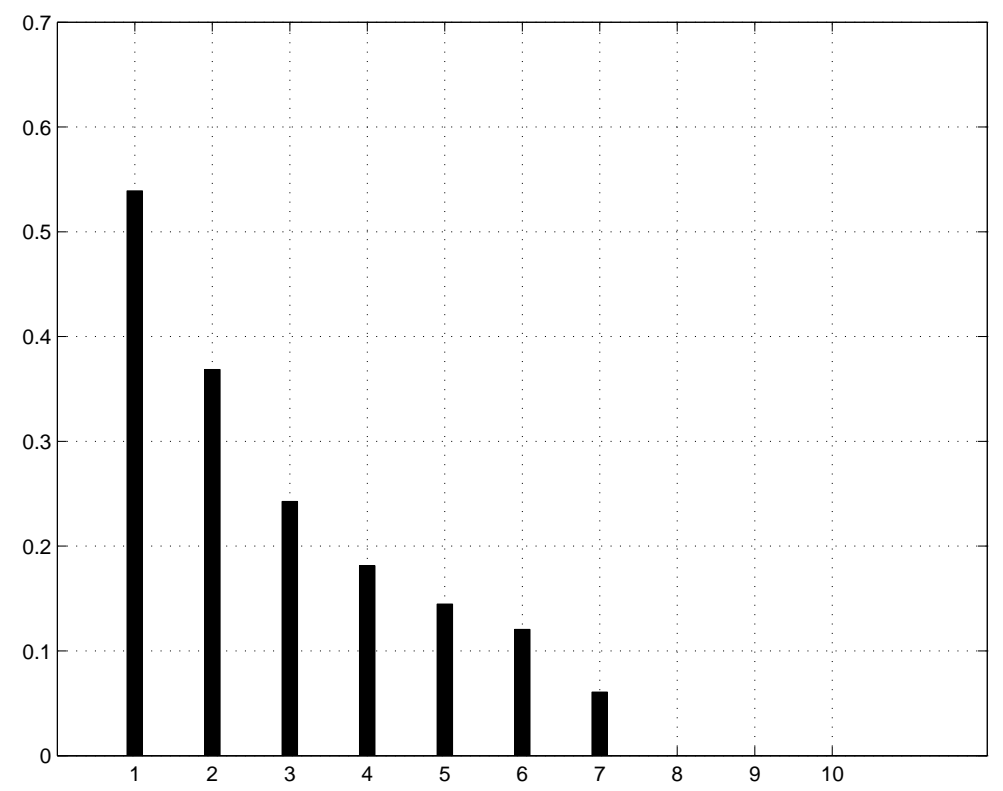

FIG. 3. Rates for 10 uniformly spaced receivers with $S N R$ density $=50 \mathrm{~m}^{2}$.

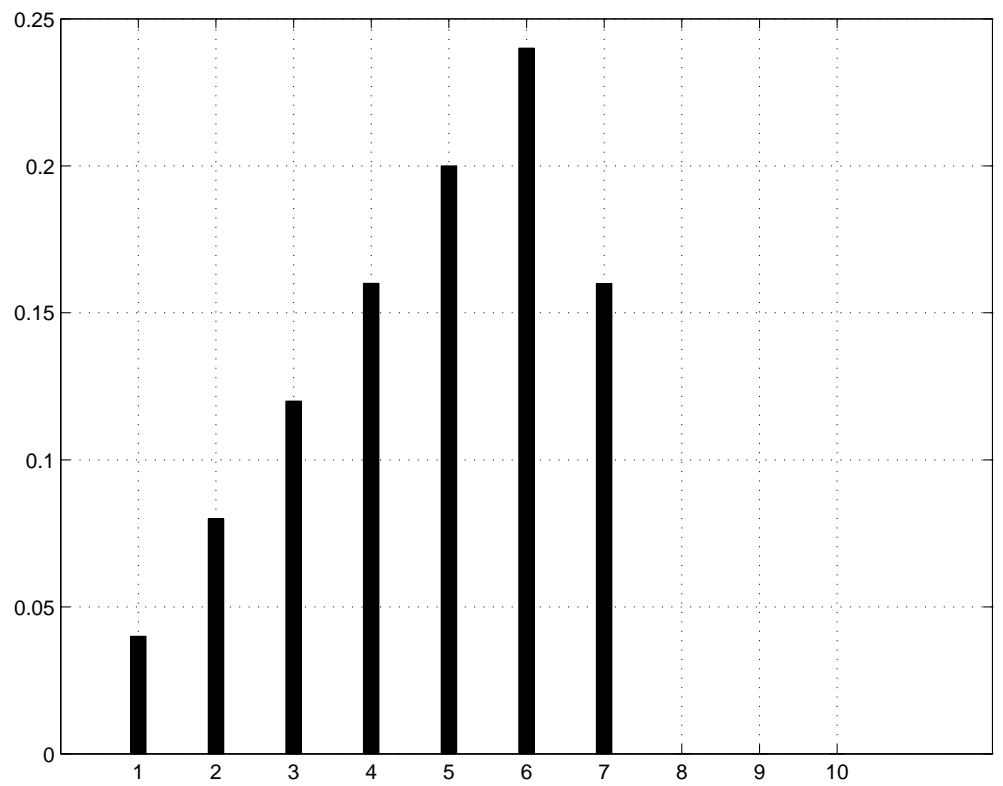

FIG. 4. Power allocation for 10 uniformly spaced receivers with $S N R$ density $=50 \mathrm{~m}^{2}$. 


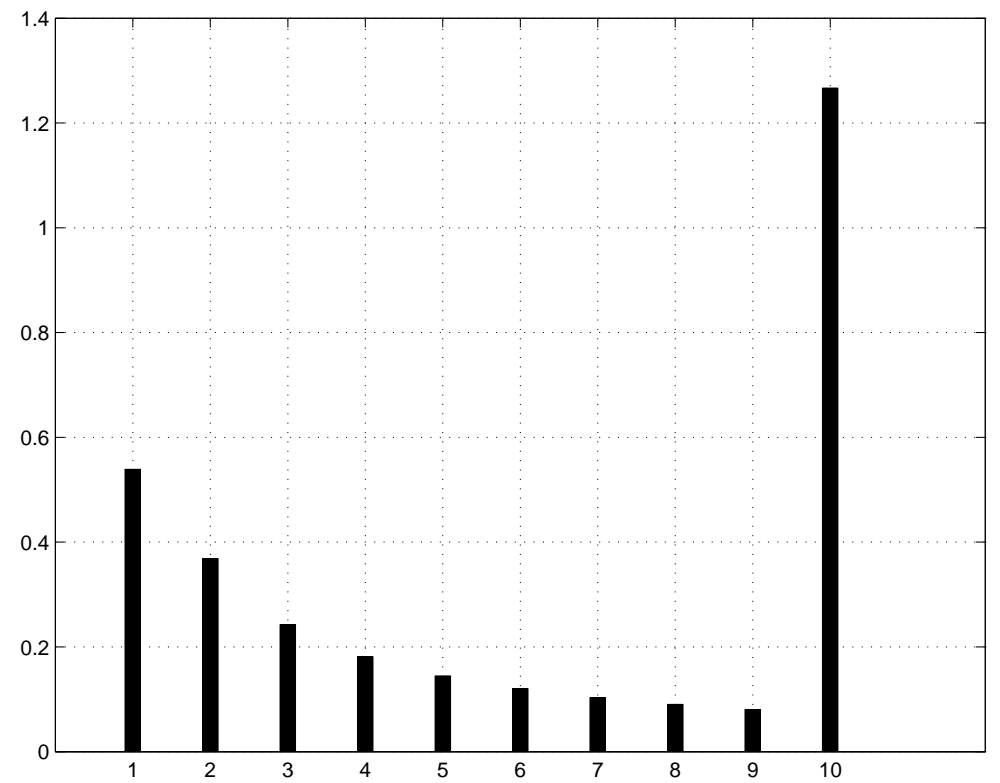

FIG. 5. Rates for 10 uniformly spaced receivers with $S N R$ density $=1000 \mathrm{~m}^{2}$.

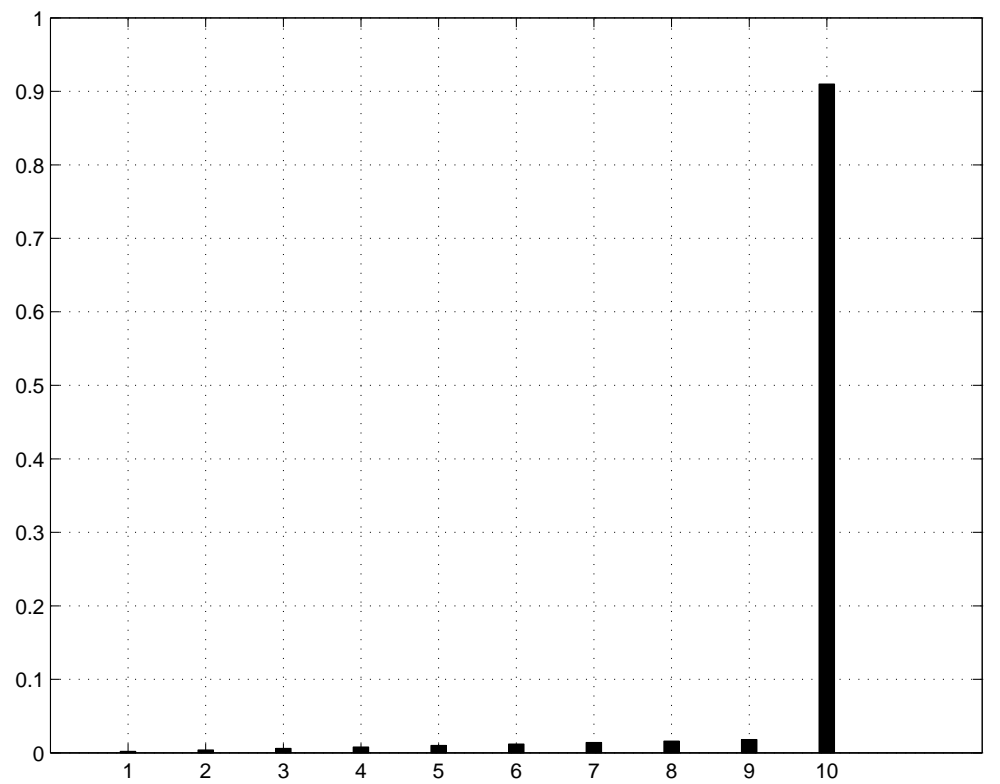

FIG. 6. Power allocation for 10 uniformly spaced receivers with $S N R$ density $=1000 \mathrm{~m}^{2}$. 


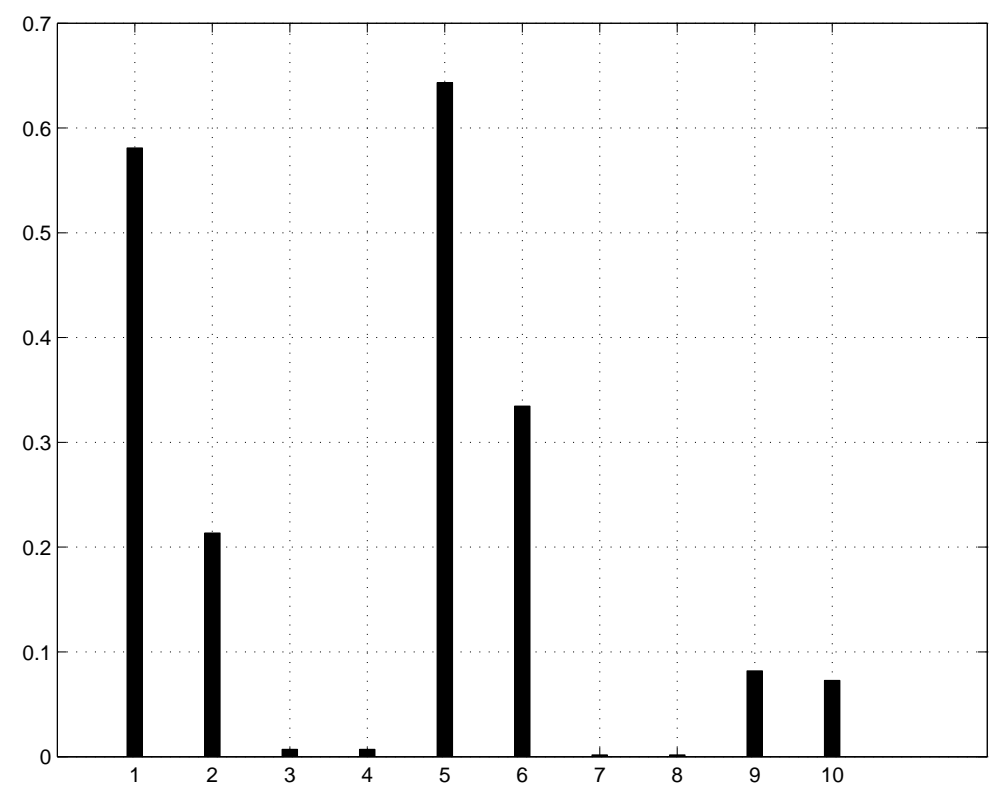

FIG. 7. Rates for 10 "bunched up" receivers with $S N R$ density $=100 \mathrm{~m}^{2}$.

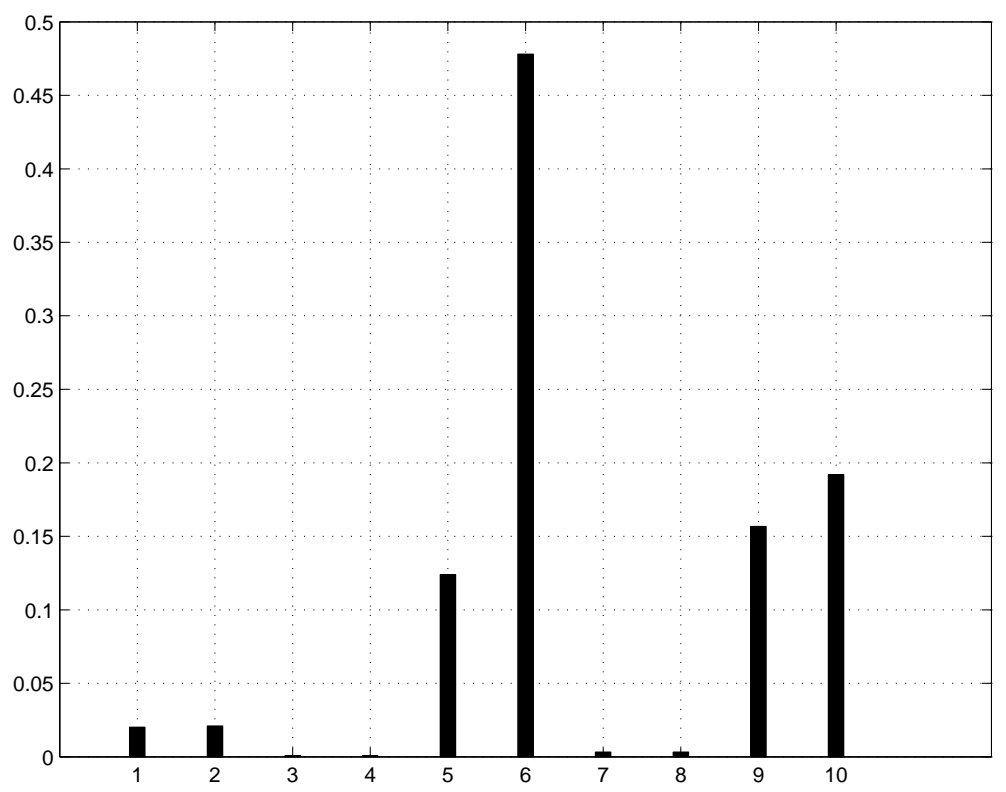

FIG. 8. Power allocation for 10 "bunched up" receivers with $S N R$ density $=100 \mathrm{~m}^{2}$. 


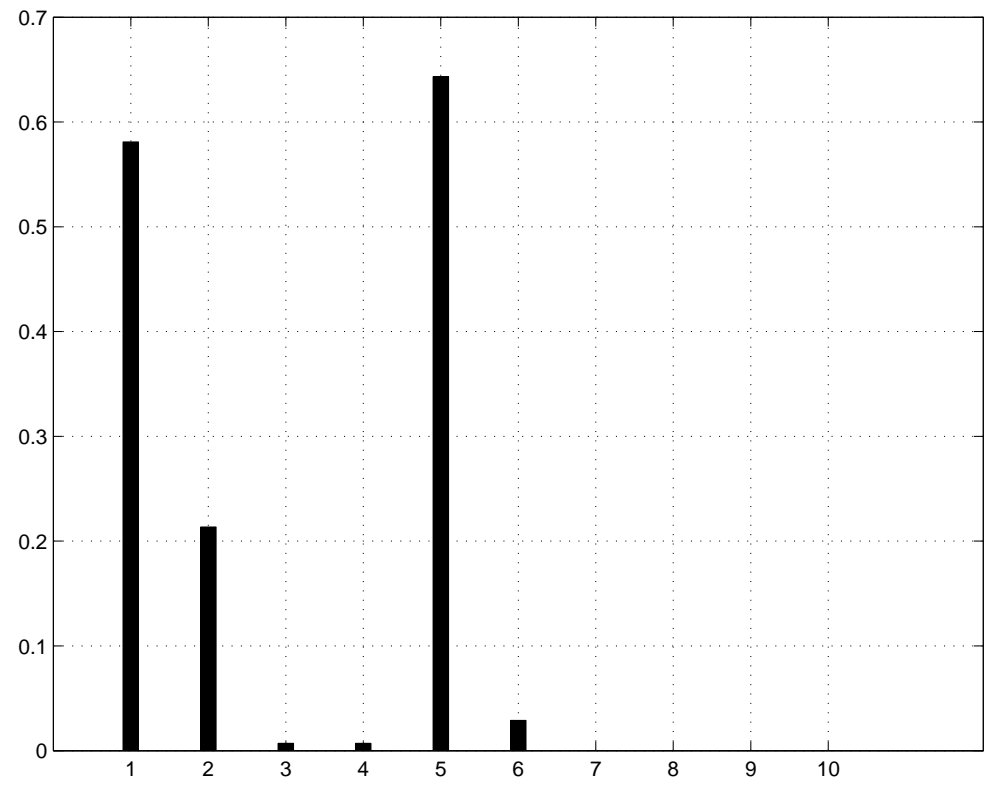

FIG. 9. Rates for 10 "bunched up" receivers with $S N R$ density $=20 \mathrm{~m}^{2}$.

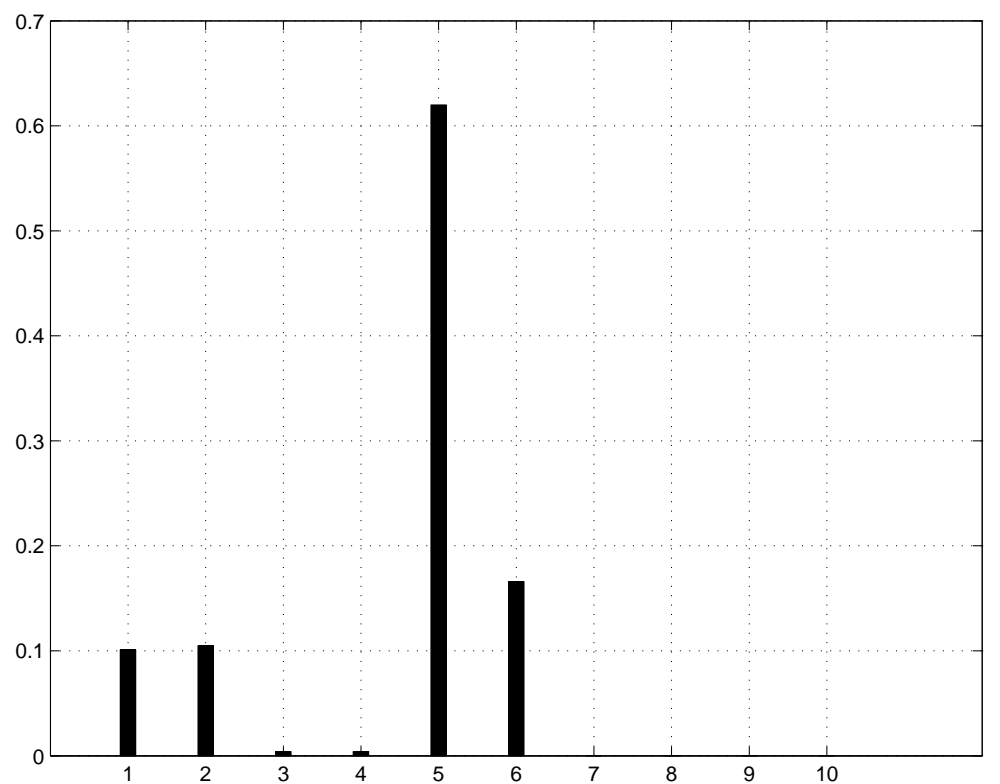

FIG. 10. Power allocation for 10 "bunched up" receivers with $S N R$ density $=20 \mathrm{~m}^{2}$. 


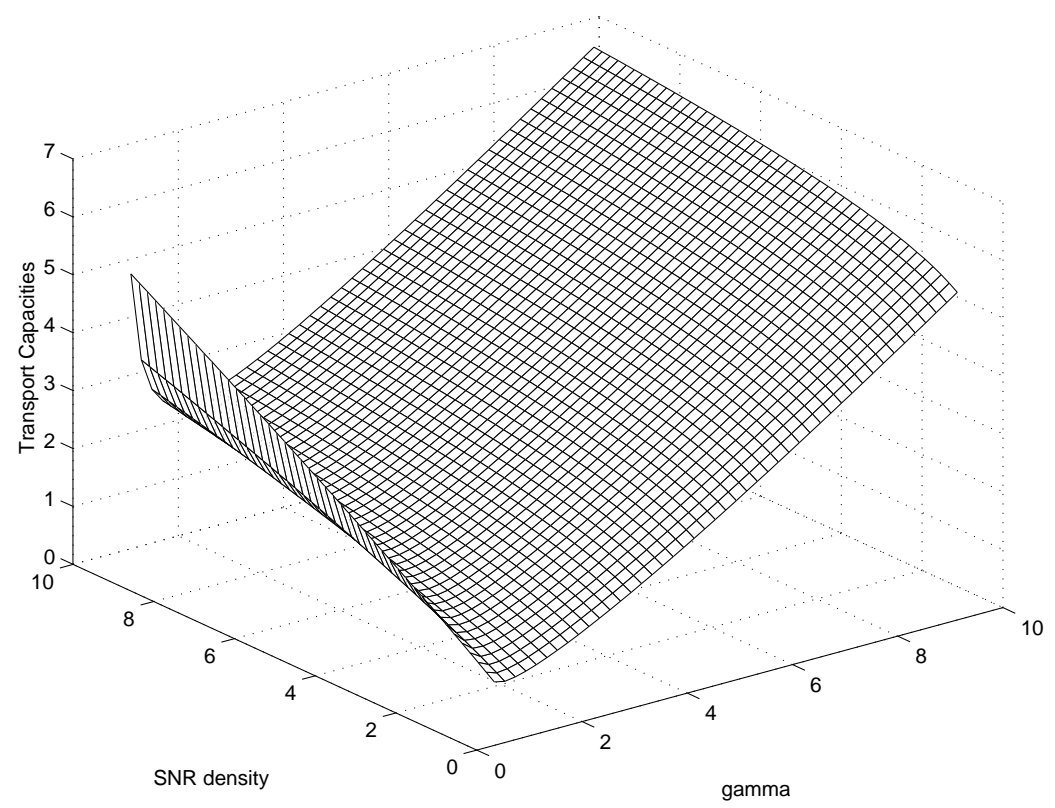

FIG. 11. Optimal transport capacity for optimal communication scheme.

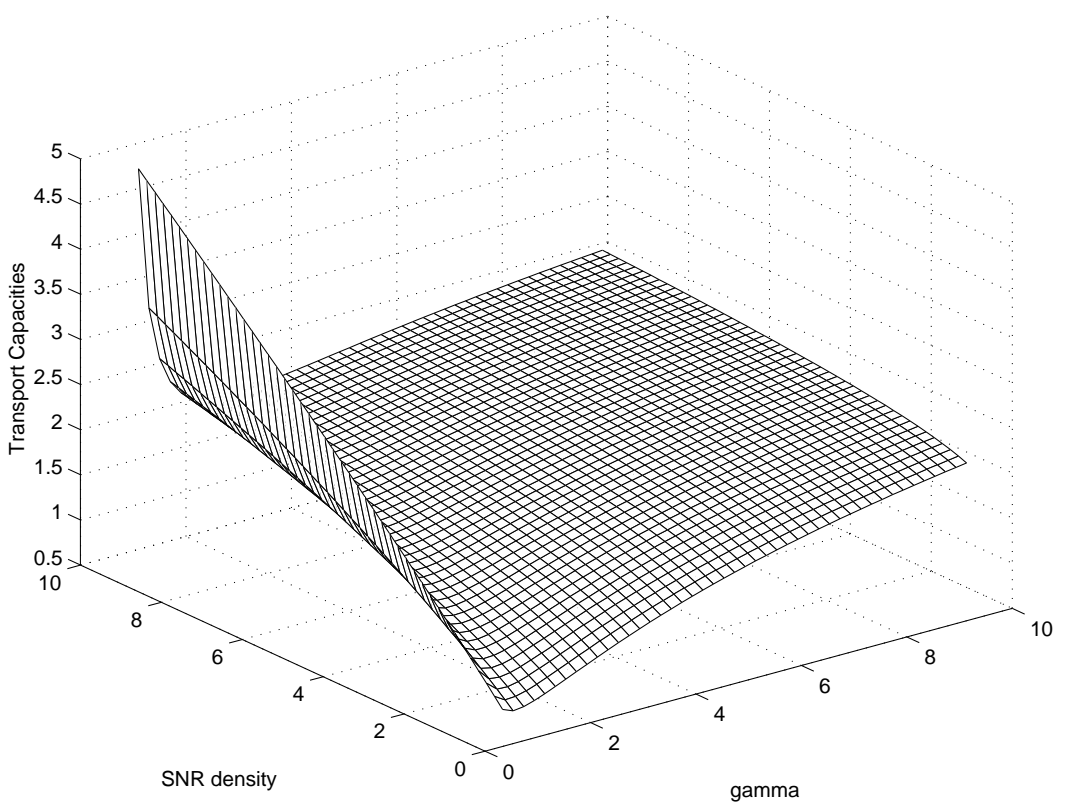

FIG. 12. Optimal transport capacity for optimal communication scheme - modified channel 


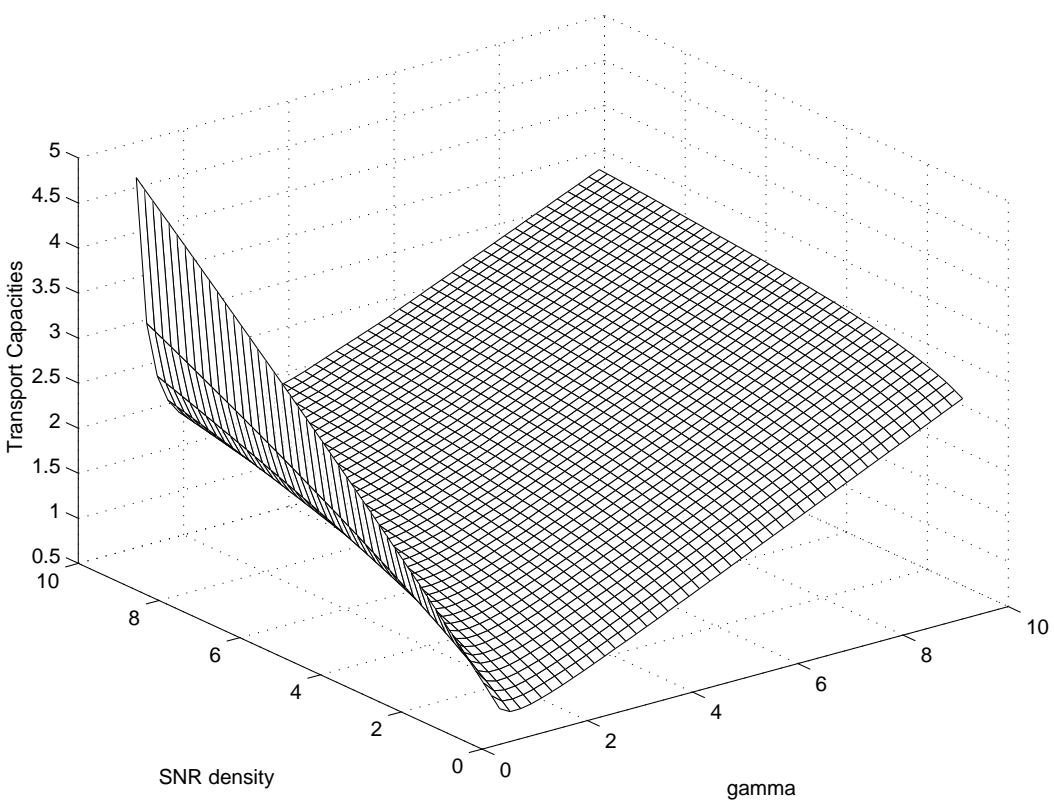

FIG. 13. Optimal transport capacity for TDMA.

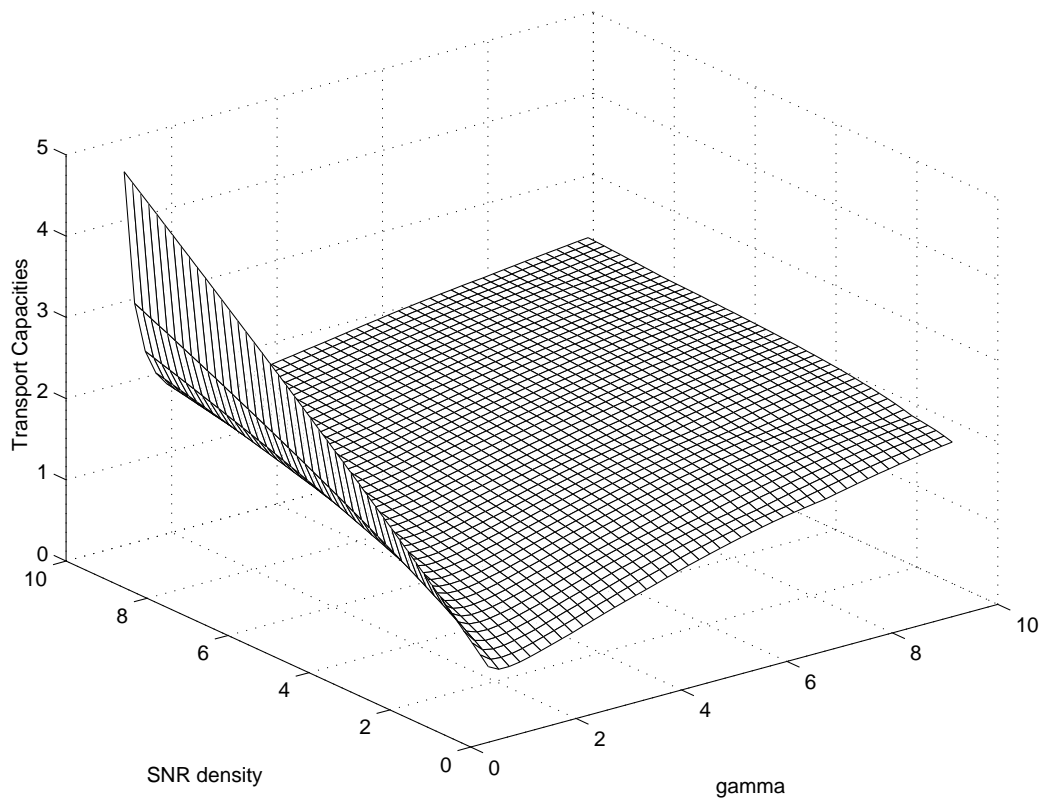

FIG. 14. Optimal transport capacity for TDMA - modified channel 